\title{
Taxonomic and biogeographic revision of the genus Lamellitettigodes (Orthoptera: Tetrigidae) with description of two new species and additional notes on Lamellitettix, Probolotettix, and Scelimena
}

JOSEF TUMBRINCK'

1 Auf der Hees 1, D-41849 Wassenberg, Germany

Corresponding author: Josef Tumbrinck (j.tumbrinck@t-online.de)

Academic editor: Daniel Petit | Received 18 March 2019 | Accepted 29 April 2019 | Published 3 September 2019

http://zoobank.org/CA0FD29D-1EE9-4176-9E1F-2EACBFFB68F1

Citation: Tumbrinck J (2019) Taxonomic and biogeographic revision of the genus Lamellitettigodes (Orthoptera: Tetrigidae) with description of two new species and additional notes on Lamellitettix, Probolotettix, and Scelimena. Journal of Orthoptera Research 28(2): 167-180. https://doi.org/10.3897/ jor.28.34605

\begin{abstract}
The genus Lamellitettigodes Günther, 1939 from Southeast Asia is reviewed. The genus currently includes seven species and is transferred to Tetriginae Rambur, 1838. Two new species are described: Lamellitettigodes novaeguineae sp. nov. from New Guinea and Lamellitettigodes karwinkeli sp. nov. from Yunnan, People's Republic of China. Lamellitettigodes palawanicus Günther, 1939 stat. nov. is no longer regarded as a subspecies of $L$. contractus, but a separate species. Two species are transferred from Euparatettix Hancock, 1904 to Lamellitettigodes: Lamellitettigodes sagittatus (Bolívar, 1887) comb. nov. and Lamellitettigodes cultratus (Bolívar, 1898) comb. nov. One species is transferred from Tetrix Latreille, 1802 to Lamellitettigodes Lamellitettigodes signatus (Bolívar, 1887) comb. nov. Xistra tricristata sumatrana Bolívar, 1898 syn. nov., Xistra tricristata Bolívar, 1898 syn. nov., and Probolotettix corticolus Blackith \& Blackith, 1987 syn. nov. are synonymized with Lamellitettigodes contractus (Bolívar, 1887). Probolotettix kevani Blackith \& Blackith, 1987 syn. nov. is synonymized with Euparatettix personatus (Bolívar, 1887). Additionally, in the genus Lamellitettix Hancock, 1904, Lamellitettix acutus Hancock, 1904 stat. rev. is retransferred from a subspecies of Lamellitettix gallinaceus (Stål, 1877) to a separate species status.
\end{abstract}

\section{Keywords}

China, fauna, Metrodorinae, New Guinea, Southeast Asia, synonymy

\section{Introduction}

At the end of August 2019, there were 2031 known Tetrigidae species worldwide (Cigliano et al. 2019). They occur on all the continents and inhabit almost all climatic zones from taiga to rainforests (Tumbrinck and Skejo 2017). Tetrigidae can easily be identified by their pronotum, which typically extends far over the body. This feature is clearly unique and proves the allocation of the species without doubt. Since 2014, the Tetrigidae of New Guinea has been revised in several papers (Tumbrinck 2014a, b, 2015, 2018, Tumbrinck and Skejo 2017). At the beginning of the revision, 67 species were known from New Guinea and the adjacent islands Aru, Biak, Waigeo, and Yapen. With the genus Lamellitettigodes, another genus is comprehensively revised within the context of the ongoing revision of the Tetrigidae of New Guinea. The genus was established by Günther (1939) and comprised two species with three subspecies. After 1939, for the first time the genus is fundamentally revised. With the addition of a new species described in this paper, there are currently 145 species known for New Guinea and the adjacent islands.

\section{Material and methods}

All specimens originate from the collections of several museums. Various cameras using various lenses were used to take photos, all in macro mode by using a stacking system with an integrated scale bar or with a macro lens and millimeter paper. No post-processing of photographs was done. Millimeter paper was placed close to the photographed specimen and subsequently used to construct a scale bar, included in the photograph, after which the millimeter paper was deleted. The morphological terminology and measurement methods follow Tumbrinck (2014a) and good taxonomic practice suggested in Lehmann et al. (2017). For many localities, the geographical coordinates were identified as accurately as possible by localization of the place found on the labels with different gazetteers and extracted using the Google Earth program. These localities are presented in square brackets.

\section{Depository abbreviations}

ANIC Australian National Insect Collection, CSIRO, Canberra City, Australian Capital Territory, Australia

ANSP Academy of Natural Sciences, Philadelphia, Pennsylvania, USA

BMEC Bohart Museum Entomology Collection, Davis, California, USA

BMNH The Natural History Museum, formerly British Museum (Natural History), London, UK

BPBM Bernice P. Bishop Museum, Honolulu, Hawaii, USA

CDS Collection Silva, Viçosa, Brasil

CJT Collection Tumbrinck, Wassenberg, Germany

CMKT Collection Tan, Singapore 
IRSNB Institut Royal des Sciences Naturelles de Belgique, Bruxelles, Belgium

LEMQ Lyman Entomological Museum and Research Laboratory, Quebec, Canada

MHNG Muséum d'Histoire Naturelle, Geneva, Switzerland

MLU Martin-Luther-Universität, Zoologisches Institut, Halle, Germany

MNCN Museo Nacional de Ciencias Naturales, Madrid, Spain MNSL Naturkundemuseum, Leipzig, Germany

MSNG Museo Civico di Storia Naturale "Giacomo Doria", Genova, Italy

MZB Museum Zoologicum Bogoriense, Bogor, Java, Indonesia

NHRS Naturhistoriska Riksmuseet, Stockholm, Sweden

NMEG Naturkundemuseum Erfurt, Germany

NMI National Museum of Ireland, Dublin, Ireland

NMW Naturhistorisches Museum Wien, Austria

OSF Orthoptera Species File Database (http://orthoptera. speciesfile.org)

RMNH Naturalis Biodiversity Center, Leiden, The Netherlands SDEI Senckenberg Deutsches Entomologisches Institut, Müncheberg, Germany

SMTD Staatliches Museum für Tierkunde, Leipzig, Germany

ZFMK Zoologisches Forschungsmuseum Alexander Koenig, Bonn, Germany

ZMHU Zoologisches Museum der Humboldt Universität, currently Museum für Naturkunde der Humboldt-Universität zu Berlin, Berlin, Germany

ZMUC Universitets København, Zoologisk Museum, København, Denmark

\section{Type specimens abbreviations}

Holotype (HT); lectotype (LT); paralectotype (PLT); paratype (PT); syntype (ST).

\section{Results}

Taxonomy

Genus Lamellitettigodes Günther, 1939

Lamellitettigodes Günther, 1939: 123; Yin et al. 1996: 878; Otte 1997: 46 .

Type species.-Paratettix contractus (Bolívar, 1887), by original designation.

Taxonomic placement and justification.-Günther (1939) assigned the genus Lamellitettigodes to the subfamily of Metrodorinae because of the lateral lobes: they are almost, but not completely, attached as in Paratettix Bolívar, 1887. This was the main character used when the subfamily was established by Bolívar (1887), and Günther also adopted it. Today, typically Metrodorinae are mainly characterized by having the median ocellus and the antenna placed below the eyes, a relatively small divergence of the rami of the frontal costa not forming wide scutellum, and a similar length of the first and third segments of the hind tarsus (PavónGonzalo et al. 2012). Many species of Metrodorinae also exhibit the posterior angles of the lateral lobes of the pronotum produced outwards, often becoming acutely spinose. These characters taken together separate the subfamily from the other eight subfamilies of Tetrigidae, although no single character is enough to characterize Metrodorinae by itself (Tumbrinck and Skejo 2017).

In contrast to Metrodorinae, in Lamellitettigodes the antenna is inserted at the lower margin of the eyes, and the first segment of the hind tarsus is longer than the third segment. The similarity of Lamellitettigodes species with Paratettix and Euparatettix is clear when comparing all morphological characters from most of the Paratettix and Euparatettix species of Southeast Asia. Lamellitettigodes species have typical characters of Tetriginae: rounded lateral lobes of pronotum close to pronotum (directed downwards, slightly sidewards; this character is somewhat similar to Metrodorinae), presence of posthumeral spots on the pronotum, L-shaped carinae of the vertex, pulvilli of the hind tarsus with apical teeth (absent in some Lamellitettigodes species), and hind wings exceeding the tip of the pronotum. Therefore, Lamellitettigodes is very close to Tetriginae and did not belong to Metrodorinae: I transfer this genus to the subfamily of Tetriginae.

Description.-Günther (1939) gave a detailed description of the genus, which is no longer applicable in some of its parts and must be amended. An improved redescription based on his description is presented here.

Body of moderate size, slender. Head and pronotum smooth. Head and eye in lateral view not at all or slightly elevated above the pronotal discus. Antenna more than 1.5 times longer than fore femur, dorsal margin of antennal groove a little bit above the ventral margin of eye, between the eyes. Eyes small and in lateral view blunt and indistinctly conoidal. Antenna filiform, 14-segmented in male (including scapus and pedicel), 15-segmented in female. Fastigium of vertex in dorsal view as broad as or a little wider than the eye, fastigium in frontal view between the eyes slightly depressed. Fastigium in dorsal view with well-developed medial, lateral carinae, and fossula. Lateral carina clearly elevated, in lateral view weakly visible above the eyes and short, in dorsal view not converging towards the front, almost parallel, bending over at right angles to the medial carina (L-shaped). Transverse carina of the vertex in dorsal view slightly convex or straight. Frontal costa and facial carina in lateral view visible in front of eye (except in L. novaeguineae). Tip of fastigium and its frontal costa in lateral view protuberant. Facial carinae in lateral view concave in front of eye and slightly convex in front of antenna. Last segments of maxillary palps not foliaceous. Anterior margin of pronotum truncated. Pronotum slightly between shoulders. Prozonal carina and median carina clear, slightly elevated (lamellate in $L$. cultratus and L. novaeguineae), interhumeral carina absent, internal lateral carinae not clearly visible. Pronotum behind shoulders weakly depressed on both sides of median carina and here very often provided with black spots (posthumeral spots) on each side. Lateral lobes broadly rounded, not as close to body as in Paratettix. Tegmen almost as long as the fore femur, rather broad and at the end broadly rounded. Macropterous and macropronotal. Alae clearly exceed the pronotum apex. Hind femora with very small antegenicular and especially genicular teeth, with some sharply tuberculate raised slants of their middle outer surface. Posterior tibia distally not or slightly widened, with a few small spines on upper edges. First segment of the hind tarsus not longer than the third, pulvilli acute, in three species with apical teeth (L. sagittatus, L. signatus, $L$. karwinkeli). The coloration is highly variable and not a useful feature for either generic or specific diagnosis (as reported for Tetriginae in Lehmann et al. 2017). 
Diagnosis. - The genus Lamellitettigodes is characterized by a protuberant tip of fastigium and frontal costa (in lateral view); facial carinae in lateral view visible and clearly concave in front of eye and slightly convex in front of antenna. Only in L. novaeguineae is tip of pronotum not visible in lateral view, but present. Additional characters for the genus are: 1) lateral carina of the vertex in frontal view clearly elevated as small fastigial horns, but in lateral view weakly visible above the eyes (Plate $5 \mathrm{Q}, \mathrm{R}, \mathrm{V}, \mathrm{W}$ ); 2 ) median carina continuous to the tip of the pronotum; 3 ) clearly keeled or lamellate median carina; and 4) keeled and more or less converging prozonal carinae.

The genus is close to Euparatettix and Paratettix (see above). In the typical representatives of mentioned genera, the tip of the fastigium is not protruding, but more or less rounded. Also, frontal horns are absent. In Paratettix the median carina is absent in the frontal margin of pronotum. A similarity exists also with Lamellitettix Hancock, 1904. The type species of the genus is Lamellitettix acutus Hancock, 1904 stat. nov. Blackith (1988) synonymized L. acutus and L. pluricarinatus Hancock, 1909 with Lamellitettix insularis (Bolívar, 1887), and L. insularis and L. fletcheri Hancock, 1915 with Lamellitettix gallinaceus (Stål, 1877) without giving evidence for the synonymy (Blackith 1992). Types of L. gallinaceus were examined by the author in NHRS and those of $L$. pluricarinatus in UMO. $L$. acutus and L. pluricarinatus are rather different from L. gallinaceus by the form of the pronotum, but it remains uncertain whether $L$. acutus and L. pluricarinatus are separate species. Further studies of the type of $L$. acutus and more specimens are needed. Lamellitettix, with its genotype L. acutus, is different from Lamellitettigodes by the tip of the fastigium which is, in lateral view, not protuberant, by the lateral carinae that are curved in dorsal view forward towards the medial carina and are not parallel, and by the lateral lobes that are acutely pointed sidewards. A similarity exists also to Xistra Bolívar, 1887 and Xistrella Bolívar, 1909. In the typical representatives of Xistra and Xistrella, the antenna is inserted in a considerable distance below the ventral margin of the eye. In Lamellitettigodes, the antenna is inserted as high as the ventral margin of the eye (Plate 5Q-X).

Composition and distribution.-Altogether seven species are now assigned to the genus Lamellitettigodes: one hitherto described species (widely distributed L. contractus from peninsular Malaysia, Singapore, Sumatra, Java, Borneo, Sulawesi, and Solomon Isl.) supplemented with a subspecies (L. c. palawanicus inhabiting Palawan archipelago of the Philippines) here elevated to the species level, two new combinations (widely distributed L. sagittatus from Vietnam, Thailand, peninsular Malaysia, Sumatra, Java, Borneo, Philippines, Moluccas Isl., Sulawesi, New Guinea, and Timor, and L. signatus from the Philippines) and two species described here as new to science (L. novaeguineae inhabiting SE New Guinea, and $L$. karwinkeli inhabiting Yunnan, PR China). One hitherto described species (L. sumatrana) and a subspecies (L. contractus tricristatus) are synonymized with $L$. contractus.

\section{Key to species of Lamellitettigodes}

1 Median carina in frontal part of pronotum compressed and elevated (Plate 1D) ............................... cultratus (Bolívar, 1898) comb. nov. Median carina in frontal part of pronotum not compressed and elevated (Plates 1A-C, 2E, H).

2 Distance between upper margin of paired ocelli and frontal margin of fastigium in frontal view less than a diameter of an ocellus (Fig. 1A); bifurcation of the frontal costa into facial carinae near frontal

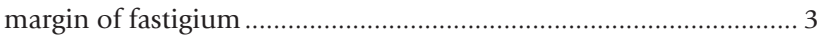
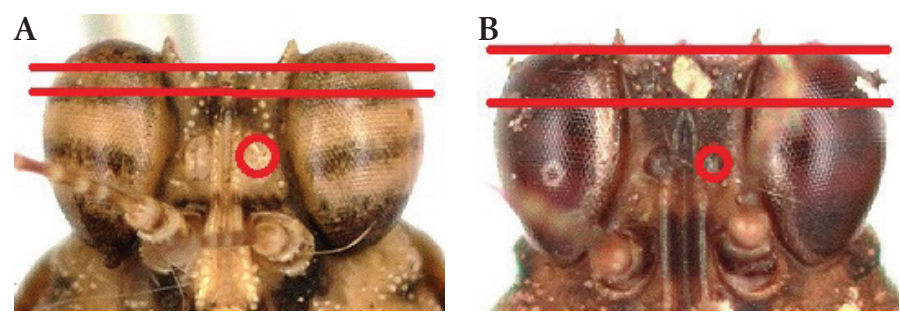

Fig. 1. Comparision of frontal costa between A. L. novaeguineae $q$ HT and B. L. palawanicus + NT.

Distance between upper margin of paired ocelli and frontal margin of fastigium in frontal view significantly larger than a diameter of an ocellus (Fig. 1B); bifurcation of the frontal costa into facial carinae in frontal view near ocelli or in the middle between ocelli and frontal margin of fastigium.

... 6

3 Pronotum, in lateral view, more or less flattened (Plate 1B, C) ....... 4 Pronotum, in lateral view, undulated (Plate 2F-H) ......................... 5 Median carina in lateral view continuously bent to tip of pronotum (Plate 1C) ................................. signatus (Bolívar, 1887) comb. nov. Median carina, in lateral view, not continuously bent to tip of pronotum; in prozona rising again (Plates $1 \mathrm{~B}, 2 \mathrm{H}$ )......

..L. sagittatus (Bolívar, 1887) comb. nov.

5 Median carina in lateral view slightly undulated but without depression above beginning of tegmen (Plate 1B).......

L. novaeguineae sp. nov.
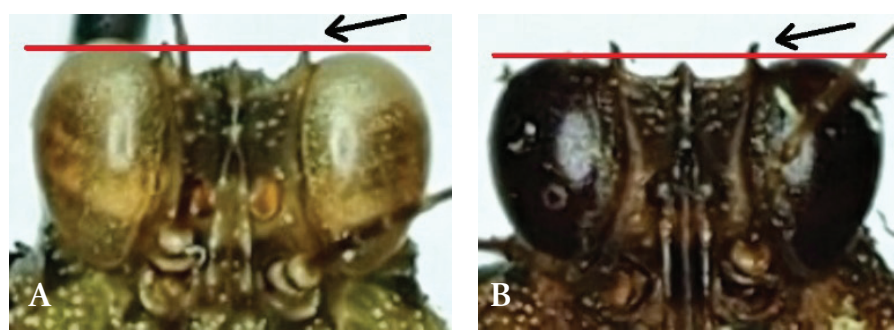

Fig. 2. Comparision of fastigial horns between A. L. contractus 9 NT from Sumatra and B. L. palawanicus $\circ$ NT.

Median carina in lateral view with depression above beginning of tegmen (Plate 2H) ............................................ karwinkeli sp. nov. Fastigial horns in frontal and lateral view not or slightly visible above eyes (Fig. 2A) ……................................ L. contractus (Bolívar, 1887) Fastigial horns in frontal and lateral view clearly visible above eyes (Fig. 2B) …............................ palawanicus Günther, 1939, stat. nov.

\section{Lamellitettigodes contractus (Bolívar, 1887)} Plates 1A, 3I, 5Q

Paratettix contractus Bolívar, 1887: 188, 272, 281; Hancock 1907a: 56; Kirby 1910: 33; Bruner 1915: 54; Günther 1935: 262-263; Günther 1937: 136; Günther 1939: 124.

Tetrix contractus: Hancock 1907b: 236, 239; Hancock 1909: 412; Hancock 1913: 52; Günther 1939: 124.

Lamellitettigodes contractus: Günther 1939: 124-125; Steinmann 1970a: 228; Blackith 1992: 100; Paris 1994: 235; Yin et al. 1996:

878; Otte 1997: 46.

Type material._LT Lamellitettigodes contractus (here designated): 온 [Indonesia, Borneo], MNCN (Cat. Tipos No 243), original label 


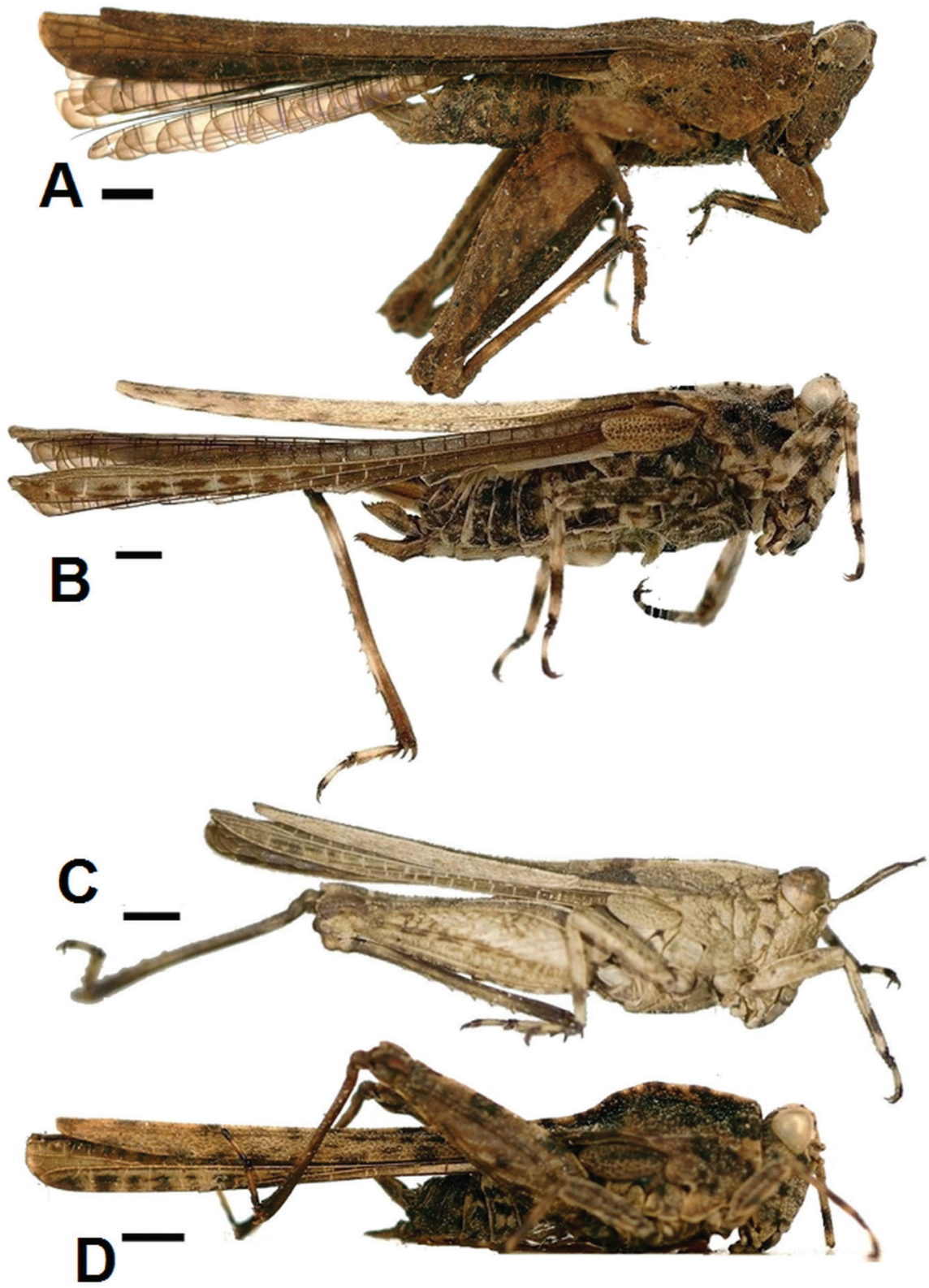

Plate 1. A-D (lateral view). A. Lamellitettigodes contractus, $q$ HT; B. Lamellitettigodes sagittatus, $q$ HT; C. Lamellitettigodes signatus, $q$ HT; D. Lamellitettigodes cultratus, o $\mathrm{HT}$. Scale bars: $1 \mathrm{~mm}$.

from Bolívar "Paratettix contractus Bol." and label "Sintipo ?" from Mercedes Paris.

PLT Lamellitettigodes contractus (here designated): + , Philippines, NHRS (NRW-ORTH 0013201).

Lamellitettigodes tricristatus (Bolívar, 1898), syn. nov.

Xistra tricristata Bolívar, 1898: 75-76; Hancock 1907a: 46; Kirby 1910: 27; Willemse 1930: 30; Willemse 1931: 44; Günther 1939: 125.

Paratettix tricristatus: Günther 1935: 263.

Lamellitettigodes contractus tricristatus: Günther 1939: 125; Steinmann 1970a: 228; Paris 1994: 251

Lamellitettigodes tricristatus: Otte 1997: 46.

Tetrix cuspidata Hancock, 1907 (Synonym)

Tetrix cuspidatus Hancock 1907b: 239-240; Hancock 1909: 413; Günther 1935: 263; Günther 1939: 125; Blackith 1992: 100.
Acrydium cuspidata: Kirby 1910: 579.

Type material._LT Lamellitettigodes tricristatus (here designated): o, [Indonesia], Java, 1893, leg. H. Fruhstorfer, MNCN (Cat. Tipos No 39)

PLTs Lamellitettigodes tricristatus (here designated): $2 \hat{\jmath} \hat{\sigma}+3 q+$, [Indonesia], Java, 1893, leg. H. Fruhstorfer, MNCN (Cat. Tipos No 40-44); $\hat{\text {, }}$ [Indonesia], Java, Sakabumi, 1893, leg. H. Fruhstorfer, IRSNB.

Notes.-Bolivar (1898) described the species under the section Metrodorae as belonging to Xistra, while earlier he described $L$. contractus within the section Tettigiae, assigned to the genus Paratettix. The two sections were distinguished by morphology of the lateral lobes-projected sidewards in Metrodorae, directed downwards in Tettigiae. When comparing types, I did not see differences in this feature. 
Günther (1939) regarded L. contractus tricristatus as a subspecies of L. contractus, distinguished from the nominal by smaller size, sharper keeled prozonal carinae and, in lateral view, clearly visible fastigial horns (above the eyes). L. c. tricristatus should be slightly smaller and should have wavy margins of the fore legs (Günther 1935). All these features are, however, in the range of variation of the species and are not of diagnostic value. Moreover, I did not find reported differences when comparing the types. Otte (1997) gave the specific status to the subspecies only with the reason of occurrence on different islands.

All these data prove that L. tricristatus (Bolívar, 1898) is conspecific with $L$. contractus.

\section{Synonym Tetrix cuspidata Hancock, 1907}

Notes.-Günther (1935) also synonymized Tetrix cuspidata Hancock, 1907 with $L$. contractus, but without giving detailed information. I have seen the STs and agree with his decision.

Type material._LT Tetrix cuspidata (here designated): ㅇ, [Indonesia], Java, Pengalangan, 4000 ft, 1893, leg. Fruhstorfer (Type 727 1/3).

PLTs Tetrix cuspidata (here designated): $2 \hat{\jmath} \hat{\jmath}$, [Indonesia], Java, Pengalangan, $4000 \mathrm{ft}, 1893$, leg. Fruhstorfer (Type 727 2/3+3/3).

Hancock refers to four specimens from the same locality but in UMO only three specimens are present.

\section{Lamellitettigodes sumatrana (Bolívar, 1898), syn. nov.}

Xistra tricristata var. sumatrana Bolívar, 1898: 76; Willemse 1928: 4; Willemse 1930: 30-31; Günther 1935: 262-263; Günther 1939: 124; Paris 1994: 250-251.

Xistra tricristata sumatrana: Hancock 1907a: 46. Xistra sumatrana: Steinmann 1970a: 230.

Xistra tricristata sumatrensis: Blackith 1992: 100. Lamellitettigodes sumatrana: Otte 1997: 46.

Type material._LT Lamellitettigodes sumatrana (here designated): ㅇ, Indonesia, Sumatra, Si-Rambé, XII.1890-III.1891, leg. E. Modigliani, MNCN (Cat. Tipos No 68), original label from Bolívar "Xistra var. sumatrana Bol." and label "Sintipo" from Mercedes Paris.

PLTs Lamellitettigodes sumatrana (here designated): $\hat{\sigma}, q^{\prime}$, [Indonesia], Sumatra, Si-Rambé, XII.1890-III.1891, leg. E. Modigliani,

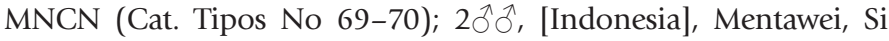
Oban, IV-VIII.1894, leg. E. Modigliani, MNCN (Cat. Tipos No 71,

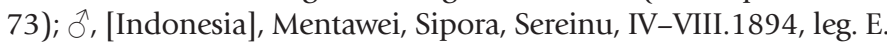
Modigliani, MNCN (Cat. Tipos No 74); +, [Indonesia], Eng[g]ano, Bua-Bua, V-VI.1891, leg. E. Modigliani, MNCN (Cat. Tipos No 72); 7 specimens, [Indonesia], Sumatra, Si-Rambé, XII.1890-III.1891, leg. E. Modigliani, MSNG [not seen]; 5 specimens, [Indonesia], Mentawei, IV-VIII.1894, leg. E. Modigliani, MSNG [not seen]; 2 specimens, [Indonesia], Eng[g]ano, Bua-Bua, V-VI.1891, leg. E. Modigliani, MSNG [not seen]; 1 specimen, [Indonesia], Nias, leg. E. Modigliani, MSNG [not seen]; $2 \hat{\jmath}, 2$, + , [Indonesia], Sumatra, SiRambé, XII.1890-III.1891, leg. E. Modigliani, IRSNB [not seen]; ㅇ, [Indonesia], Mentawei, Sipora, Sereinu, V-VI.1894, leg. E. Modigliani, RMNH; ڤ̂̃, [Indonesia], Mentawei, Si Oban, IV-VIII.1894, leg. E. Modigliani; +, [Indonesia], Sumatra, Si-Rambé, XII.1890-III.1891, leg. E. Modigliani, NHRS (NRM-ORTH 0013047 + 0013046).

Notes.-Bolívar (1898) described the taxon as a variety of Xistra tricristata. In contrast to L. tricristatus, the prozonal carinae are not curved to the middle and not keeled. The margins of the fore legs are slightly undulated. Günther (1935) synonymized Xistra tricristata var. sumatrana with Paratettix contractus without giving details. According to Günther (1939), the specimens of L. sumatrana have in lateral view only slightly visible elevated lateral carinae above the eyes and wavy median carina in front of and behind the shoulders. In the specimens of $L$. contractus from Borneo, these undulations are weaker. Otte (1997) gave the subspecies a specific status based on occurrence on different islands (as in L. tricristatus mentioned above). All these features are within the range of variation of the species and do not represent diagnostic traits. I did not find differences when comparing the types and, therefore, L. sumatrana (Bolívar, 1898) (original combination Xistra tricristata sumatrana) is regarded as conspecific with L. contractus.

\section{Probolotettix corticolus Blackith \& Blackith, 1987, syn. nov.}

Probolotettix corticolus: Blackith and Blackith 1987: 5-8; Blackith 1990: 89-90; Blackith 1992: 146; Yin et al. 1996: 902 (error: carticolus); Otte 1997: 57.

Type material.-HT Probolotettix corticolus: + , Indonesia, Sulawesi, Minehassa Prov., Dumoga-Bone National Park, 13.III.1985, leg. Blackith, RMNH (not seen, probably lost).

PTs Probolotettix corticolus: $\hat{\sigma}$, Indonesia, Sulawesi Utara, Dumoga-Bone National Park, I.-III.1985, leg. R. \& R. Blackith, $\mathrm{BMNH}$; + , Indonesia, Sulawesi Utara, Dumoga-Bone National Park, I.-III.1985, leg. R. \& R. Blackith, LEMQ; $\widehat{\jmath}+q$, Indonesia, Sulawesi Utara, Dumoga-Bone National Park, I.-III.1985, leg. R. \& R. Blackith, NMI (not seen), MZB (not seen).

Notes.-After the description and the drawing of the HT in Blackith and Blackith (1987), this species is a typical L. contractus. I have seen the PT in BMNH and LEMQ and there is no doubt that they are L. contractus.

Additional material examined.-Philippines: $3 \partial^{\lambda}{ }^{\lambda}$, Siargao, $9^{\circ} 52^{\prime} 33^{\prime \prime} \mathrm{N}$, $126^{\circ} 00^{\prime} 35^{\prime \prime E}$, on leaf, 28 m, 7.IV.2018, leg. M. K. Tan, Yeo, H., Yap, S. A. \& Baroga, J. B., CMTK. Indonesia: +, Sumatra, Lac de Toba, 1.V.1929, leg. Prince Leopold, RMNH; , Sumatra, Soekaranda, leg. Dohrn, SMTD; +, W. Sumatra, Padang Panjang, 28.VIII.1991, leg. W. Guidetti, CJT; 9 , Sumatra, Sibolangit, 550 m, 11.X.1921, leg. J. B.

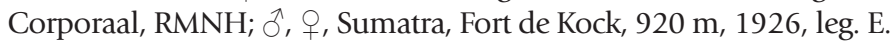
Jacobson, RMNH; $\hat{\jmath}$, Sumatra, Lubuksikaping (Sumatra's Westkust), $450 \mathrm{~m}, 1926$, leg. E. Jacobson, RMNH; $2 \delta^{\lambda} \delta^{\lambda}, 7$ 우우, Sumatra, S. W. Lampong distr., Mt. Tanggamoes, Gisting ult., 500 m, XII.1939, leg. M. A. Lieftinck, RMNH; 2§̂̋, Mentawai, Siberoet, 24.+28.IX.1924, leg. H. H. Karny, RMNH; $\widehat{\text { }}$, Mentawai, Sipora, 11.X.1924, leg. H. H. Karny, RMNH; , Nias, Sisobahili I., Tankec, ca. 14 km v. Gunung Sitoli, IX.1991, leg. W. Guidetti, CJT; §, o , Westjava, Mt. Guntur, Garoet, 1350 m, leg. Overbeck; §̂, ᄋ, Java, G. Tjikorai, 900 m, X.1934, leg. E. Jacobson, RMNH; $\widehat{\partial}$, Java, Tjikadjang, Bandjarwangi, W. Java, 800900 m, 7.-10.IV.1939, leg. M. A. Lieftinck, RMNH; ふ઼, Java, Penandjoeng Bay, Kalinnoetjang, 300 m, VII.1936, leg. M. A. Lieftinck, RMNH; $3 \hat{\jmath} \widehat{\partial}, 4$ 우으, Borneo, Midden O. Borneo, 19.VIII.1925, RMNH, leg. H. C. Siebers;, , Borneo, Tabang, Bengen River, East Borneo, 125 m, 10.IX.1956, leg. A. M. R. Wegner, RMNH; ㅇ, Borneo, O. Borneo, Tandjong Redeb, leg. Mjöberg, NHRS; ㅇ, Borneo, Sarawak Distr., leg. Mjöberg, NHRS; $3 \hat{\jmath} \widehat{\jmath}, 2$ 우, Borneo, Long Navang, leg. Mjöberg, NHRS; $2 \hat{\jmath}, 3$ 우, Borneo, Kajan River, leg. Mjöberg, NHRS; 9 , Borneo, East-Sabah, Sepilok RDC, 587'57.57"N, 117 ${ }^{\circ} 94^{\prime} 75.76 " \mathrm{E}$, leg. T. Kirschey, CJT. Solomon Islands: $2 \hat{\jmath} \widehat{\partial}$, Malaita, Auki, 2-20 m, 22.IX.1957, leg. J. L. Gressitt, BPBM. 
Differential diagnosis. - L contractus has more or less parallel prozonal carinae, slightly bent backwards. The distance between the upper margin of the superior ocelli and frontal margin of the fastigium is significantly larger than a diameter of an ocellus in frontal view. The species is similar to L. palawanicus. This species can be easily separated by the tip of fastigium and frontal costa, which are distinctly protuberant in front of the eyes, and the fastigial horns, which are clearly visible above the eyes.

Measurements._-(in mm) L. c. contractus (LT/PLT): Pronotum length 12.61/11.63; pronotum lobe width 3.25/2.92; pronotum height $2.53 / 1.58$; vertex width $0.54 / 0.45$; eye width $0.45 / 0.45$; tegmen length $1.60 / 1.50$; hind wing length $11.18 / 10.88$; postfemur length LT 5.46; postfemur width LT 1.70.

L. c. tricristatus syn. nov. LT: Pronotum length 8.71 ; pronotum lobe width 2.57; pronotum height 1.95 ; vertex width 0.43 ; eye width 0.39 ; tegmen length 1.07 ; hind wing length 7.28 ; postfemur length 3.95; postfemur width 1.25.

L. c. sumatrana syn. nov. (LT/PLT): Pronotum length 10.40/10.01; pronotum lobe width 2.99/2.73; pronotum height $2.74 / 2.21$; vertex width $0.50 / 0.49$; eye width $0.41 / 0.39$; tegmen length 1.56/1.43; hind wing length $10.27 / 9.23$; postfemur length 5.07/4.55; postfemur width $1.69 / 1.43$.

T. cuspidata (LT/PLT2/3/PLT3/3): Pronotum length $10.79 / 10.92 / 8.71$; pronotum lobe width $3.25 / 2.80 / 2.65$; pronotum height $1.65 / 1.50 / 1.35$; vertex width $0.50 / 0.47 / 0.45$; eye width $0.47 / 0.47 / 0.41$; tegmen length $1.55 / 1.45 / 1.30$; hind wing length 8.97/10.53/8.58; postfemur length 5.20/4.40/4.24; postfemur width $1.65 / 1.45 / 1.35$.

Additional measurements: (in mm) o Sumatra, Lac de Toba: Pronotum length 9.75; pronotum lobe width 2.95; pronotum height 2.29 ; vertex width 0.54 ; eye width 0.41 ; tegmen length 1.30 ; hind wing length 9.23; postfemur length 4.88; postfemur width 1.45 . + , Borneo, Sepilok RDC: Pronotum length 10.79; pronotum lobe width 3.00 ; pronotum height 1.45 ; vertex width 0.47 ; eye width 0.45 ; tegmen length 1.55; hind wing length 10.27; postfemur length 4.81; postfemur width 1.60. $\hat{\sigma}$, Solomon Is., Malaita: pronotum length 9.88; pronotum lobe width 2.60; pronotum height 1.45 ; vertex width 0.47 ; eye width 0.43 ; tegmen length 1.35 ; hind wing length 8.97; postfemur length 4.42; postfemur width 1.40 .

Distribution.-The species inhabits the Southeast Asian mainland (Günther 1939), Singapore (Hancock 1909), islands of Southeast Asia - Sumatra (and adjacent Nias, Mentawai, and Enggano), Java, Borneo, Sulawesi (Günther 1939), and finally the Solomon Islands. The species has not been found hitherto in New Guinea. Hancock (1907a), however, refers to New Guinea under Xistra tricristata, and Günther (1935) wrote" (...) also known to me from SE New Guinea". I have not been able to detect this species in New Guinea and the Bismarck Archipelago, although it is found to inhabit the Solomon Islands. Hancock's (1907a) and Günther's (1935) records could belong to L. novaeguineae sp. nov.

\section{Lamellitettigodes sagittatus (Bolívar, 1887), comb. nov. Plates 1B, 3J, 5R}

Paratettix sagittatus Bolívar, 1887: 188, 280-281. Xistra sagittata: Bolívar 1898: 76; Hancock 1907a: 46; Kirby 1910: 27; Willemse 1930: 31, 207; Günther 1939: 160; Blackith 1992:

197; Paris 1994: 248; Yin et al. 1996: 930 (error: sagittaria); Otte 1997: 67
Euparatettix sagittatus: Günther 1937: 138-139; Günther 1938: 2-3, 41; Günther 1941: 155-156; Günther 1942: 345; Steinmann 1970a: 232; Yin et al. 1996: 870; Zheng et al. 2011: 385; Deng 2016: 302.

Type material._LT Lamellitettigodes sagittatus (designated by Paris 1994): + , Philippines, Daraga, MNCN (Cat. Tipos No 126), original label from Bolívar "Xistra sagittata Bol." and label "Lectotipo" from Mercedes Paris.

ST (= PLT) Lamellitettigodes sagittatus:, , Philippines, NHRS [referred by Bolívar 1887 but not found in NHRS].

Synonym Euparatettix pulvillus Hancock, 1910: 360; Günther, 1937: 131, 138.

HT Euparatettix pulvillus: Malaysia, Selangor, Riverside Estate Kwala, IX.1907, leg. H. C. Pratt, UMO.

Note: Günther (1937) synonymized E. pulvillus Hancock, 1910 with $L$. sagittatus after the description of Hancock. I have seen the HT and agree with his decision.

Synonym Tetrix polypictus Hancock, 1913: 52-53; Günther, 1937: 131, 138; Otte, 1979: 41

Acrydium polypictum: Hancock, 1915: 135.

HT Tetrix polypictus: + , Malaysia, Borneo, Kuching, ANSP (not seen).

Note: Günther (1937) synonymized T. polypictus Hancock, 1913 with L. sagittatus after the description of Hancock.

Additional material examined.-Vietnam: 3 ठึ, 5 $\uparrow+$, Bac Giang Prov., Tay Yen Tu Nat. Res. Thanh So'n, 18.-21.V.2015, leg. A. Skale, CJT, NMEG; 1 , Thai Nguyen Province, vic. Ngoc Thanh, vic. Me Linh (IEBR station), $21^{\circ} 23^{\prime} 03^{\prime \prime} \mathrm{N}, 105^{\circ} 42^{\prime} 44^{\prime \prime} \mathrm{E}, 2 . V .2012$, leg. A. Weigel, CJT.

Thailand: ㅇ, Khao Soi Dao, 15.X.1985, leg. S. Ingrisch, CJT; 우, Chanthaburi Khao Soi Dao, 12.VI.1988, leg. S. Ingrisch, CJT.

Malaysia: +, Selangor, Riverside Estate Kwala, IX.1907, leg. H. C. Pratt, UMO; $\delta^{\wedge}, 2+$, Pahang, Bukit Chitimani along overgrown path at base of limestone crop, $3^{\circ} 26^{\prime} 48^{\prime \prime} \mathrm{N}, 102^{\circ} 0^{\prime} 47^{\prime \prime} \mathrm{E}$, 12.III.2016, leg. L. Willemse, RMNH, CJT; $\widehat{\partial}$, Pahang, Bukit Chitimani along overgrown path at base of limestone crop, $3^{\circ} 26^{\prime} 48^{\prime \prime} \mathrm{N}$, $101^{\circ} 55^{\prime} 59 " \mathrm{E}, 11 . I I I .2016$, leg. L. Willemse, RMNH.

Malaysia: Borneo: ô, Banguey [Pulau Banggi], 1935, SMTD; $6 \widehat{\jmath}, 5$ 우우, East-Sabah, Sepilok RDC, 16.XI.2016, leg. T. Kirschey, CJT; $\widehat{\jmath}$,, Sabah, Kinabalu National Park, 5.-7.VIII.1984, leg. S. Ingrisch, CJT; $\widehat{\jmath}$, + , Sabah, Kinabalu National Park, 8.VIII.1984, leg. S. Ingrisch, ZFMK; + , Sarawak, Ng. Jagau, Sg. Ngemah, 3 Div., ex hill padi, single hill sample JF-2-18, 22.XI.1974, leg. D. Munroe, LEMQ.

Indonesia: Sumatra: $\widehat{\partial}$, Padang, MLU; + , Soeruil, IV.1878, ㅇ, Eng[g]ano, Bua-Bua, V.1891-VI.1891, leg. E. Modigliani, MSNG; RMNH; $\widehat{\jmath}, 4$ 우, Pangherang-Pisang, X.1890-III.1891, leg. E. Modigliani, MSNG; ㅇ, Fort de Kock, 920 m, 1925, leg. E. Jacob-

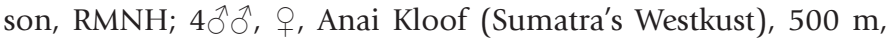
1925+1926, leg. E. Jacobson, SMTD, RMNH; 0 , ㅇ, Medan, Gedong Djohore, 7.II.1928, leg. M. Mohr, RMNH; + , S. W. Lampong distr., Mt. Tanggamoes, Gisting ult., 500 m, XII.1939, leg. M. A. Lieftinck, RMNH; + , Palembang Office, Dinas Kehutanan, [2 ${ }^{\circ} 56^{\prime} 42.245^{\prime \prime}$, $\left.104^{\circ} 43^{\prime} 44.663^{\prime \prime E}\right], 17$ m, leg. T.Kirschey, CJT;

Mentawai: + , Siberoet, 18.IX.1924, leg. H. H. Karny, RMNH;

Sulawesi: $\widehat{\text { J }, ~ N r . ~ M o r o w a l i, ~ R a n u ~ R i v e r ~ a r e a, ~ 27 . I .-20 . I V .1980, ~}$ leg. M. J. D. Brendel, BMNH; $\delta$, street Kotamobagu-Motoling (North Sulawesi), 15.-16.I.2001, leg. M. Hoffmann, MNSL; $2 ㅇ ㅜ$, Prov. Gorontalo, Taman Nasional Nani Bogani Wartabone, 7.VIII.2016, leg. T. Kirschey, CJT; 
Java: + , Salatiga, $\left[7^{\circ} 19^{\prime} \mathrm{S}, 110^{\circ} 33^{\prime} \mathrm{E}\right]$, leg. W. Roepke, RMNH; ㅇ, Tjimandala [Cimandala], XII.1923, SMTD; $2 q+q$, G. Tjikorai, X.1934, leg. E. Jacobson, RMNH; + , Buitanzorg [Bogor], 17.IX.1941, leg. P. A. Blijdorp, RMNH; ${ }^{1}$, Bogor, Botanical Garden, 21.III.1993, leg. S. Ingrisch, CJT; $4 \hat{\jmath} \widehat{\jmath}, 8$ 우우, Bogor, Botanical Garden, 6 $35^{\prime} 51^{\prime \prime S}, 106^{\circ} 47^{\prime} 58^{\prime \prime}$ E, 21.II.+31.VII.2016, leg. T. Kirschey, CJT; + , Surabaya $43 \mathrm{~km} \mathrm{SW}$, Mt. Pennggungan, hotel PPLH, $370 \mathrm{~m}, 7^{\circ} 36^{\prime} 29^{\prime \prime} \mathrm{S}, 112^{\circ} 09^{\prime} 40^{\prime \prime} \mathrm{E}, 18 .-19 . V I I I .2017$, leg. D. Telnov, NMEG;

Borneo: 今̂, Tandjong Redeb, leg. Mjöberg, NHRS;

Moluccas: + , Buru, Nal Beti, 10.V.1921, leg. L. J. Toxopeus, RMNH; 옹 Isl. Batjan, Salawaku river, 50-100m, 17.VI.1963, $\mathrm{RMNH}$;

Aru: + , Aru, Wokan, 1873, leg. O. Beccari, MSNG;

Waigeo: $3 \hat{\jmath} \hat{\sigma}, 4$ 우, $3 \mathrm{~km}$ W Waisai, 40-50 m, 026'04"S, $130^{\circ} 47^{\prime} 41$ "E, 18.II.2012, leg. D. Telnov, CJT, NMEG;

West Papua: $\delta$, $q$, Cyclops Mts., Sabron, Camp 2 [2³0'S, $\left.140^{\circ} 25^{\prime} \mathrm{E}\right], 2000 \mathrm{ft}, \mathrm{VI} .1936$, leg. L. E. Cheesman, BMNH; +, Cyclops Mts., Sabron, Camp 1 [2³0'S, $\left.140^{\circ} 25^{\prime} \mathrm{E}\right], 1200$ ft, 13.V.1936, leg. L. E. Cheesman, BMNH;, , Cyclops Mts., Sabron, Camp 1 [2 $\left.{ }^{\circ} 30^{\prime} \mathrm{S}, 140^{\circ} 25^{\prime} \mathrm{E}\right], 9300 \mathrm{ft}, \mathrm{VI} .1936$, leg. L. E. Cheesman, BMNH; ㅇ, Cyclops Mts., Jayapura, Sentani, [2³6'S, 140 $\left.37^{\prime} \mathrm{E}\right], 100 \mathrm{~m}$, 15.VI.1959, leg. J. L. Gressitt, BPBM; $3 \circ+$, Waris, S. of Hollandia [Jayapura], [3 ${ }^{\circ} 11^{\prime} \mathrm{S}, 140^{\circ} 53$ 'E], 450-500 m, 8.-15.VIII.1959, leg. T. C. Maa, CJT; $2 \hat{\jmath}$, Doberai Peninsula, Arfak mts., Anggi Gigi Lake S env., Uper vill., $2200 \mathrm{~m}, 1^{\circ} 18^{\prime} 05^{\prime \prime} \mathrm{S}, 133^{\circ} 54^{\prime} 24^{\prime \prime} \mathrm{E}, 10 .-11$. IX.2015, leg. D. Telnov, CJT;

Papua New Guinea: $\hat{\partial}$, Standlager bei Malu, [4²13'S, $\left.142^{\circ} 49^{\prime} \mathrm{E}\right]$, leg. S. G. Bürgers, III.-IV.1912, ZMHU; ô, Kokoda, 1200 ft, VIII.1933, leg. L. E. Cheesman, SMTD; §̂, Kokoda, 1200 ft, VII.1933, leg. L. E. Cheesman, BMNH; ふૈ, Orrori, 3500 ft, VII.1933, leg. L. E. Cheesman, BMNH; $\widehat{\jmath}$, Upper Jimmy Valley, Wum, [5³4'S, $\left.144^{\circ} 35^{\prime} \mathrm{E}\right], 840 \mathrm{~m}, 18 . \mathrm{VII} .1955$, leg. J. L.

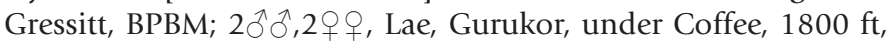
7.VII.1957, leg. J. H. Ardley, ANIC; $2 \circ+$, Gurukor, Wampi Valley, under Coffee, $\left[6^{\circ} 49^{\prime} \mathrm{S}, 146^{\circ} 37^{\prime} \mathrm{E}\right], 3000 \mathrm{ft}, 7 . \mathrm{VII} .1957$, leg. J. H. Ardley, ANIC; 2 우, Maprik [3 $\left.{ }^{\circ} 39^{\prime} S, 143^{\circ} 03^{\prime} \mathrm{E}\right], 18 . X .1957$, leg. J. Smart, BMNH; + , Bokondini, $40 \mathrm{~km} \mathrm{~N}$ of Baliem Val., 1300 m, 5.-11.XI.1961, leg. S. \& L. Quate, BPBM; 2 우, Bulo-

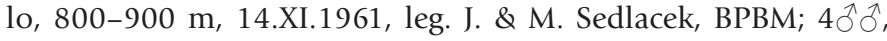

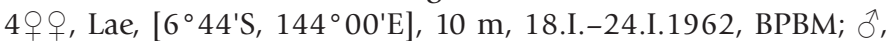
Keria, Amazon Bay area, 1650 ft, 29.VI.-22.VII.1962, leg. W. W. Brandt, ANIC; + , Sum-Sum, $64 \mathrm{~km} \mathrm{~N}$. of Wau, 15.II.1963, leg. H. W. Clissold, ВРВM; 2 우, Wau, [7²0'S, $\left.146^{\circ} 43^{\prime} \mathrm{E}\right], 1200 \mathrm{~m}$, 15.IV.1963, leg. J. Sedlacek, BPBM; Ō, 7.V.1963, Ambunti, Sepik River, $200 \mathrm{~m}$, leg. R. Straatman, BРBM; , Northern district, Managalese area, VIII.1964, leg. R. Pullen, ANIC; $0^{2}$, Popondetta, [8 $\left.{ }^{\circ} 46^{\prime} \mathrm{S}, 148^{\circ} 14^{\prime} \mathrm{E}\right], 25 \mathrm{~m}, \mathrm{VI} .1966$, leg. Shanahan \& Lippert, BPBM; 9 , Markham R. to Gabensis Village near Lae, 30.V.1967,

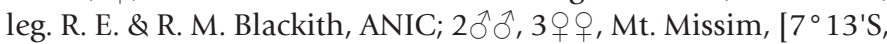
$\left.146^{\circ} 49^{\prime} \mathrm{E}\right], 1800 \mathrm{~m}, 20$. VII.1969, leg. J. L. Gressitt \& Y. Hirashima, BPBM; đ̃, 2 우, Morobe Prov., Wau, W.E.I., 22.V + 25.V.1982, leg.

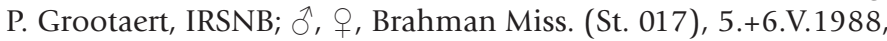
leg. J. van Stalle, IRSNB; $\hat{\sigma}$, Madang Prov., Bundi [5 $5^{\circ} 43^{\prime} \mathrm{S}$, $145^{\circ} 13^{\prime}$ E], 8.V.-10.V.1988, leg. J. van Stalle, IRSNB.

Differential diagnosis. - L. sagittatus has, together with $L$. signatus and L. karwinkeli, pulvilli of the hind tarsi bearing apical teeth. Bifurcation of the frontal costa into facial carinae is close to the transverse carinae of the vertex. In L. signatus, the median carina is bent to the tip of the pronotum while in L. sagittatus and L. karwinkeli the median carina rises again in the prozona. In contrast to $L$. karwinkeli, the median carina of L. sagittatus is flat or weakly wavy. In frontal view, there is a right angle between lateral and transverse carina in L. karwinkeli, while in L. sagittatus the angle is rounded.

Notes.-Günther (1937) retransferred this species to Euparatettix, with the note "although certainly related to Lamellitettigodes". Günther wrote in 1939 (translation): "I tend to think that the real affinity [of Lamellitettigodes] at least to Euparatettix sagittatus Bol. exists; but this species represents a true Paratettix or Euparatettix, and is to be left in these genera." L. sagittatus corresponds much better to the genus characteristics of Lamellitettigodes than those of Euparatettix or Paratettix. The carinae are clearly keeled. The prozonal carinae are the same as in L. contractus, and the tip of the fastigium is clearly protuberant in lateral view. I have examined the HT of Euparatettix pulvillus and agree with the synonymization by Günther (1937). I have not examined the type of Tetrix polypictus and was not able to check the synonymization by Günther (1937).

Specimens of $L$. sagittatus have variable size and coloration. Specimens from New Guinea have in frontal view less flattened fastigium than specimens from other regions. However, they belong to L. sagittatus because all other characteristics are the same.

Measurements._(in mm) HT: Pronotum length 11.05; pronotum lobe width 3.00 ; pronotum height 2.07; vertex width 0.43 ; eye width 0.50 ; tegmen length 1.50 ; hind wing length 11.18 ; postfemur length 5.20; postfemur width 1.65.

E. pulvillus HT: Pronotum length 12.22; pronotum lobe width 3.19; pronotum height 3.37 ; vertex width 0.56 ; eye width 0.56 ; tegmen length 1.59; hind wing length 12.35; postfemur length 5.60; postfemur width 1.80 .

Distribution.-The species inhabits Vietnam, Thailand, peninsular Malaysia, Sumatra and adjacent islands (Enggano, Mentawai), Java, Borneo, the Philippines, Moluccas Isl. (= whole Southeast Asia after Günther 1938), New Guinea (with adjacent islands Aru, Waigeo), and Timor (Günther 1937).

\section{Lamellitettigodes signatus (Bolívar, 1887), comb. nov. Plates $1 \mathrm{C}, 3 \mathrm{~K}, 5 \mathrm{~S}$}

Tettix signatus Bolívar, 1887: 268.

Acrydium signatum: Kirby 1910: 45.

Tetrix signatus: Bruner 1915: 54; Blackith 1992: 185; Paris 1994: 249; Otte, 1997: 131.

Tetrix signata: Steinmann 1970a: 233; Yin et al. 1996: 920.

Type material.-HT (designated as LT by Mercedes Paris 1994) Lamellitettigodes signatus: + , Philippines, [Prov. Eastern Samar], Dolores, MNCN (Cat. Tipos No 123), original label from Bolívar "T. signatus Bol." and label "Lectotipo" from Mercedes Paris.

Additional material examined.-Indonesia: ㅇ, Indonesia, Ins. Buru, leg. H. Kühne, NMW.

Differential diagnosis. - L. signatus is unique in having median carina extended in a small flattened arch to the frontal margin of pronotum. Other characters are the shorter pronotum and hind wings, but possibly in other species there are specimens with shortened pronotum and hind wings that have not been found yet.

Measurements._(in mm) HT: Pronotum length 10.89; pronotum lobe width 3.00 ; pronotum height 2.54 ; vertex width 0.66 ; eye 


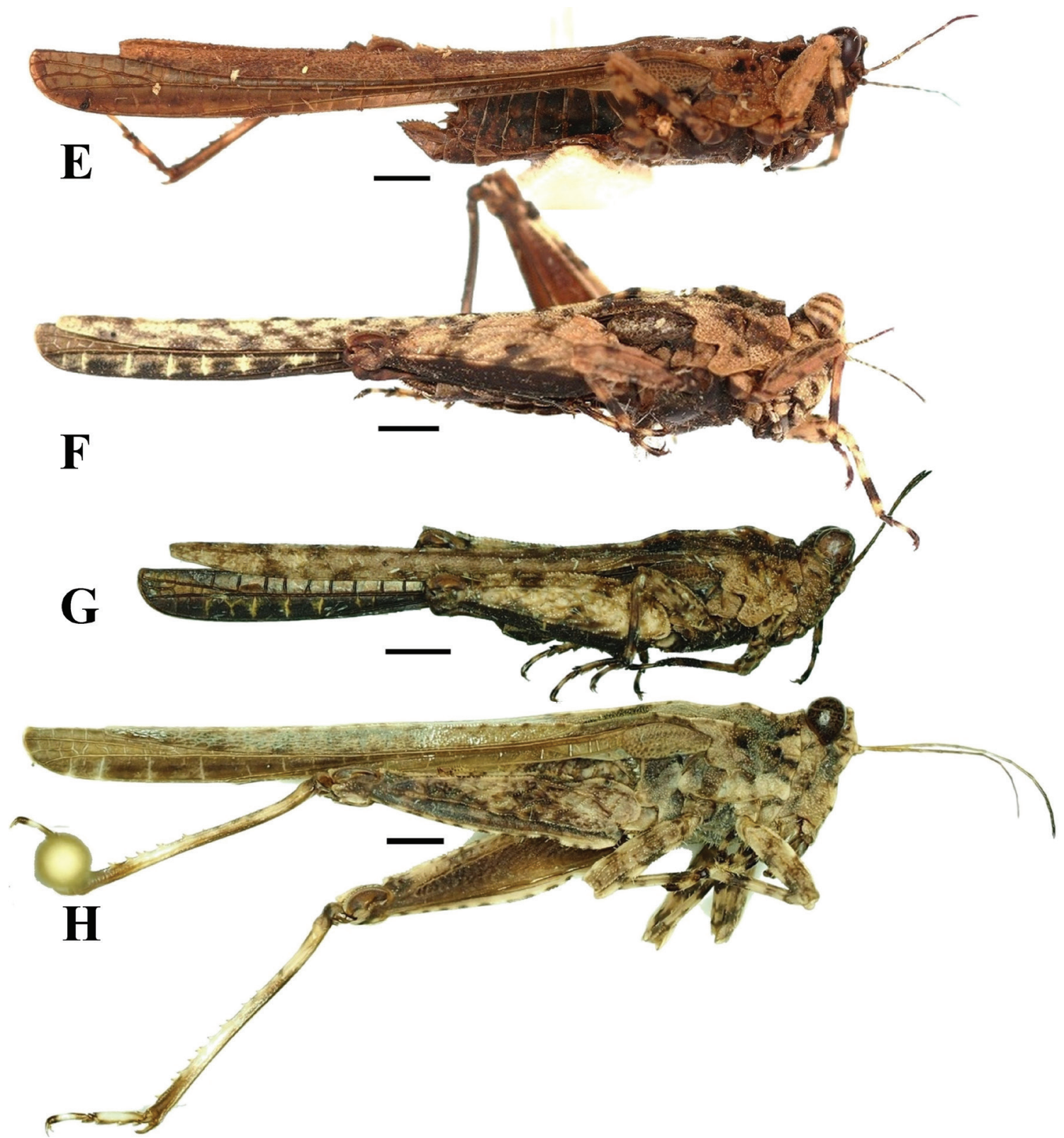

Plate 2. E-H (lateral view). E. Lamellitettigodes palawanicus (SMTD), ; F. Lamellitettigodes novaeguineae, ㅇ HT; G. Lamellitettigodes novaeguineae, ô PT 9/12; H. Lamellitettigodes karwinkeli, o HT. Scale bars: $1 \mathrm{~mm}$.

width 0.55; tegmen length 1.54; hind wing length 9.94; postfemur length 6.69; postfemur width 1.95 .

Distribution.-Known from the locus typicus (Dolores, Philippines) and from Buru (Maluka, Indonesia).

I identified one $q$ from Thailand (Khon Kaen, Nam Nao National Park, 24.V.1988, leg. S. Ingrisch, CJT) as Lamellitettigodes cf. signatus, but further investigations are needed to confirm this identification.

\section{Lamellitettigodes cultratus (Bolívar, 1898), comb. nov. Plates 1D, 3L, 5T}

Paratettix cultratus Bolívar, 1898: 188, 77-78; Hancock 1907: 56; Kirby 1910: 33; Günther 1936: 349; Günther 1938: 3, 41; Steinmann 1970b: 162; Paris 1994: 236; Yin et al. 1996: 895. Euparatettix cultratus: Blackith 1992: 63.
Type material.-HT Lamellitettigodes cultratus: ${ }^{\circ}$, [Indonesia, West-Papua], Andai [0 $\left.0^{\circ} 55^{\prime} \mathrm{S}, 134^{\circ} 01^{\prime} \mathrm{E}\right]$, VIII.1872, leg. L. M. D'Albertis, MSNG.

Additional material examined. - Indonesia: West Papua, Biak NE, 10 km N Bosnik, prim. Urwald, 10.II.1998, leg. A. Weigel, NMEG; 오, Biak, Kampong, Landbouw, 40 m, 25.-28.V.1958, leg. J. L. Gressitt \& T. C. Maa, ZFMK; ㅇ, Biak, Mangrowawa, 50-100 m, 29.V.1959, leg. T. C. Maa, CJT; $\hat{O}^{2}$,, , West Papua, Star Range, Sibil (op licht), [4 $\left.4^{\circ} 45^{\prime} \mathrm{S}, 140^{\circ} 40^{\prime} \mathrm{E}\right], 1260 \mathrm{~m}, 16 . V .1959$, Neth. New Guinea Exp. 1959, RMNH; 옹 West Papua, Nabire, S. Geelvink Bay, [3 ${ }^{\circ} 22^{\prime}$,

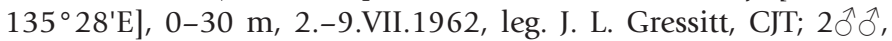
, West Papua, Nabire, S. Geelvink Bay, [ $\left.3^{\circ} 22^{\prime} \mathrm{S}, 135^{\circ} 28^{\prime} \mathrm{E}\right], 10-$ 40 m, 10.X.1962, light trap, leg. N. Wilson, CJT; $\partial^{2}$, + , West Papua, Nabire, jungle along stream, 5-50 m, 25.VIII.-2.IX.1962, leg. H. Holtmann, BPBM; 9 , West Papua, Bodem, [1 ${ }^{\circ} 58^{\prime} \mathrm{S}, 138^{\circ} 44^{\prime} \mathrm{E}$ ], 10.-17.VII.1959, leg. T. C. Maa, BPBM; + , West Papua, Bodem, 11 


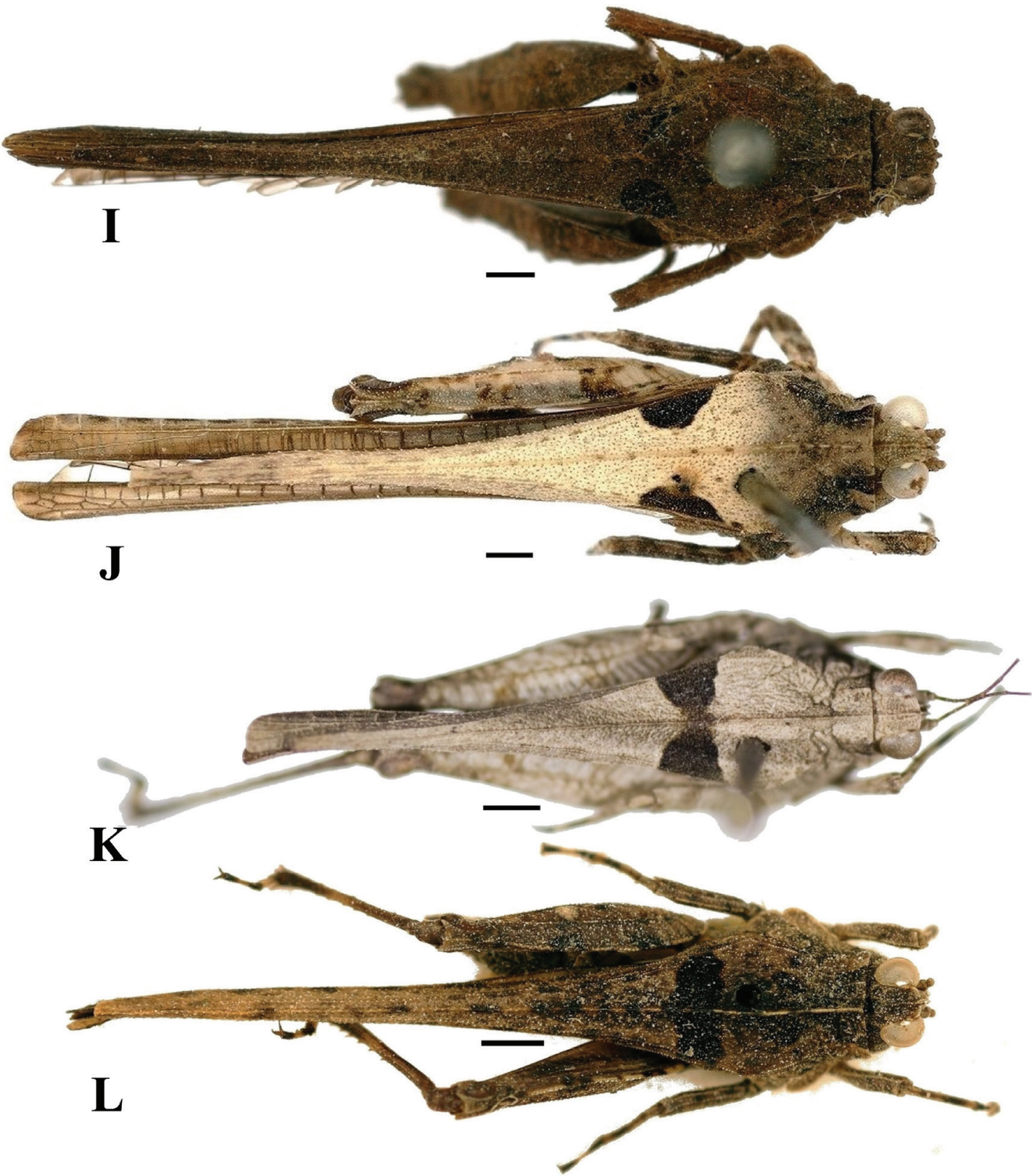

Plate 3. I-L (dorsal view). I. Lamellitettigodes contractus, + HT; J. Lamellitettigodes sagittatus, 9 HT; K. Lamellitettigodes signatus, 9 HT; L. Lamellitettigodes cultratus, + HT. Scale bars: $1 \mathrm{~mm}$.

$\mathrm{km}$ SE of Oerberfaren, [ $\left.1^{\circ} 58^{\prime} \mathrm{S}, 138^{\circ} 44^{\prime} \mathrm{E}\right], 100 \mathrm{~m}, 7 .-17 . V I I .1959$, M. V. light trap, leg. T. C. Maa, CJT; $\widehat{~}$, West Papua, Vogelkop, Manokwari, [052'S, $\left.134^{\circ} 05^{\prime} \mathrm{E}\right], 75 \mathrm{~m}, 11 . \mathrm{VIII} .1957$, leg. H. D. Elmo, CJT; $\delta^{\lambda}$, West Papua, River Tor (mouth), 4 km E of Hol Maffen,

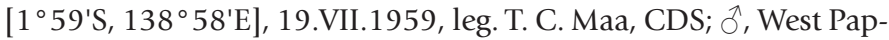
ua, Cyclops Mountains, Jayapura, Sentani, [2 $\left.{ }^{\circ} 36^{\prime} \mathrm{S}, 140^{\circ} 37^{\prime} \mathrm{E}\right], 100$ m, 15.VI.1959, leg. J. L. Gressitt, BPBM; ㅇ, West Papua, Waris, S of Hollandia, [3¹1'S, $140^{\circ} 53$ 'E], 400-450 m, 1.-7.VIII.1959, leg. T. C. Maa, CJT. Papua New Guinea: + , Gulf Prov., Lakekamu Basin, Ivimka Res. Station, $120 \mathrm{~m}, 7^{\circ} 44^{\prime} \mathrm{S}, 146^{\circ} 30^{\prime} \mathrm{E}, 18 . \mathrm{IV} .2000, \mathrm{leg}$. T. A. Sears, CJT; 2 우, [Central Prov.], Kokoda, [ $\left.8^{\circ} 39^{\prime} \mathrm{S}, 147^{\circ} 15^{\prime} \mathrm{E}\right]$, $1200 \mathrm{ft}$, VIII.1933, leg. L.E. Cheesman, BMNH \& SMTD; 2 우, [Central Prov.], Kokoda, [8³9'S, 147 $\left.{ }^{\circ} 15^{\prime} \mathrm{E}\right], 1200 \mathrm{ft}, \mathrm{VIII} .1933$, leg. L.E. Cheesman, BMNH; Õ, Orrori, 3500 ft, VII.1933, leg. L. E. Cheesman, BMNH; §larve, Plarve, [Northern Prov.], KokodaPitoki, 400 m, [855'S, $\left.147^{\circ} 44^{\prime} \mathrm{E}\right]$, 23.III.1956, leg. J. L. Gressitt,
BPBM; 9 larve, [Northern Prov.], Kokoda-Pitoki, 450 m, [855'S, $\left.147^{\circ} 44^{\prime} \mathrm{E}\right], 24 . \mathrm{III} .1956$, leg. J. L. Gressitt, CJT; 우, [Morobe Prov.], Garaina, [7053'S, $\left.147^{\circ} 08^{\prime} \mathrm{E}\right], 830 \mathrm{~m}, 13 .-15 . I .1968$, leg. J. \& M. Sedlacek, BPBM; 오 [Morobe Prov.], Garaina, [7053'S, $\left.147^{\circ} 08^{\prime} \mathrm{E}\right]$, 550-750 m, 16.I.1968, leg. J. \& M. Sedlacek, BPBM; ${ }^{\circ}$, [Western Prov.], Fly River, Olsobip, [5 $\left.{ }^{\circ} 23^{\prime} \mathrm{S}, 141^{\circ} 32^{\prime} \mathrm{E}\right], 700-1150 \mathrm{~m}$, leg. J. \& M. Sedlacek, CJT; $2+\circ$, [Western Highlands Prov.], Upper Jimmi Valley, Wum, [5³4'S, $\left.144^{\circ} 35^{\prime} \mathrm{E}\right], 840$ m, 18.VII.1955, leg. J. L. Gressitt, MNSL \& BPBM; $\widehat{\sigma}$, [Chimbu Prov.], Karimui, South of Goroka, [6³0'S, $\left.144^{\circ} 51^{\prime} \mathrm{E}\right], 1000 \mathrm{~m}, 2 . V I .1961$, leg. J. L. \& M. Gressitt, ZFMK;

q larve, New Britain, Karavat $(+20 \mathrm{~km})$, sous de vieilles souches, 300 m, 18.VII.1979, leg. J. D. Bourne, MHNG; đ’, New Britain, Gazelle Pen., Upper Warangoi, 350-600 m, 28.-29.XI.1962, leg. J. Sedlacek, CJT; + larve, New Ireland, Danu, Kalili Bay, 30.IV.1962, leg. Noona Dan Expedition 61-62, ZMUC. 
Differential diagnosis. - L. cultratus is unique in having highly lamellate and arcuate median carina above the shoulders. This character is already visible in nymphal stages. Additional unique characters are the short and strongly converging prozonal carinae and short antennae (1.3 times longer than fore femur).

Measurements.-(in mm) Pronotum length HT 12.09; pronotum lobe width 2.90; pronotum height HT 2.15; vertex width HT 0.54; eye width HT 0.47; tegmen length HT 1.50; hind wing length HT 11.18; postfemur length HT 4.70; postfemur width HT 1.45.

Distribution.-The species inhabits New Guinea, Biak, and the Bismarck Archipelago - New Britain and New Ireland.

\section{Lamellitettigodes palawanicus Günther, 1939, stat. nov. Plates 2E, 4M, 5U}

Lamellitettigodes contractus palawanicus Günther, 1939: 79-80.

Type material._LT Lamellitettigodes palawanicus (here designated): +, Philippines, N. Palawan, Binaluan, XI-XII.1913, leg. G. Boettcher, SDEI.

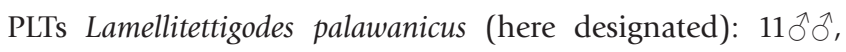
7우, Philippines, N. Palawan, Binaluan, XI-XII.1913, leg. G. Boettcher, SDEI.

Note: Günther refers to $4 \hat{\jmath} \widehat{\partial}, 15+q$ as STs.

Additional material examined. $-2 \uparrow q$, Philippines, N. Palawan, Binaluan, XI-XII.1913, leg. G. Boettcher, SMTD.

Differential diagnosis. - L. palawanicus has almost parallel prozonal carinae that are slightly bent backwards. The distance between upper margin of superior ocelli and frontal margin of the fastigium, in frontal view, is significantly larger than the diameter of ocellus. The species is similar to L. contractus. L. palawanicus can be separated from the similar species by the tip of fastigium and frontal costa that are distinctly protuberant in front of the eyes and by fastigial horns that are clearly visible above the eyes.

Measurements. - (in $\mathrm{mm})$ + , Philippines, Binaluan (SMTD): Pronotum length 11.96; pronotum lobe width 3.4 ; pronotum height 1.90; vertex width 0.50 ; eye width 0.45 ; tegmen length 1.60; hind wing length 12.74; postfemur length 6.50; postfemur width 1.95 .

Distribution.-The species is hitherto known only from Palawan (Binaluan), the Philippines.

Lamellitettigodes novaeguineae sp. nov.

http://zoobank.org/DF0F6588-4DC4-477B-9E4E-F7DC8DD56A76 Plates $2 \mathrm{~F}, \mathrm{G}, 4 \mathrm{~N}, \mathrm{O}, 5 \mathrm{~V}, \mathrm{~W}$

Material examined. - HT Lamellitettigodes novaeguineae: + , Papua New Guinea, Gulf Prov., Lakekamu Basin, Ivimka Res. Station (Malaise Trap), $7^{\circ} 44^{\prime} \mathrm{S}, 146^{\circ} 30^{\prime} \mathrm{E}, 120 \mathrm{~m}, 4$.-6.III.2000, leg. T. A. Sears, BMEC.

Type material. -PTs Lamellitettigodes novaeguineae: $\hat{0}$,,$+(1 / 12+2 / 12)$, Papua New Guinea, Gulf [Prov.], Lakekamu Basin, Ivimka Res. Station, (M[alaise $] \mathrm{T}[\mathrm{rap}]), 7^{\circ} 44^{\prime} \mathrm{S}, 146^{\circ} 30^{\prime} \mathrm{E}, 120 \mathrm{~m}, 4 .-6 . I I I .2000$, leg. T. A. Sears, BMEC (deposited in ZFMK); 3 우 (3/12-5/12), Papua New Guinea, Gulf [Prov.], Lakekamu Basin, Ivimka Res. Station, $7^{\circ} 44^{\prime} \mathrm{S}, 146^{\circ} 30^{\prime} \mathrm{E}, 120 \mathrm{~m}$, 5.III.2000, leg. T. A. Sears, BMEC; 우
(6/12), Papua New Guinea, Gulf [Prov.], Lakekamu Basin, Ivimka Res. Station, (M[alaise]T[rap]), $7^{\circ} 44^{\prime} \mathrm{S}, 146^{\circ} 30^{\prime} \mathrm{E}, 120 \mathrm{~m}, 19 .-23$. IV.2000, leg. T. A. Sears, BMEC (deposited in BMNH); ㅇ (7/12), Papua New Guinea, Gulf [Prov.], Lakekamu Basin, Ivimka Res. Station, $7^{\circ} 44^{\prime} \mathrm{S}, 146^{\circ} 30^{\prime} \mathrm{E}, 120 \mathrm{~m}, 15 .-20 . I V .2000$, leg. T. A. Sears, BMEC (deposited in ZMHU); + (8/12), Papua New Guinea, Gulf [Prov.], Lakekamu Basin, Ivimka Res. Station, (M[alaise]T[rap]), $7^{\circ} 44^{\prime} \mathrm{S}, 146^{\circ} 30^{\prime} \mathrm{E}, 120 \mathrm{~m}, 18 . \mathrm{IV} .2000$, leg. T. A. Sears, BMEC (deposited in RMNH); $\sigma^{\top}(9 / 12)$, Papua New Guinea, Gulf [Prov.], Lakekamu Basin, Ivimka Res. Station, $7^{\circ} 44^{\prime} \mathrm{S}, 146^{\circ} 30^{\prime} \mathrm{E}, 120 \mathrm{~m}$, 18.IV.2000, leg. T. A. Sears \& binatung brigade, BMEC (deposited in RMNH); ô (10/12), Papua New Guinea, Gulf [Prov.], Lakekamu Basin, Ivimka Res. Station, (M[alaise]T[rap]), $7^{\circ} 44^{\prime} \mathrm{S}, 146^{\circ} 30^{\prime} \mathrm{E}$, 120 m, 26.III.2000, leg. T. A. Sears \& binatung brigade, BMEC; 2 우 $(11 / 12+12 / 12)$, Papua New Guinea, Fly River, Kiunga, 35 m, VIII.1969, leg. J. \& M. Sedlacek, BPBM.

Diagnosis. - The species is easily recognizable by the raised median carina with broad depression between the shoulders. This depression clearly distinguishes it from L. cultratus. It is also slightly raised in Chinese L. karwinkeli but does not reach the same height and is not lamellated. As a further feature, the fastigium tip is only slightly visible in front of the eyes.

Description.-Body of moderate size and slender. Head and pronotum smooth. Head and eye in lateral view weakly elevated above the pronotal discus. Dorsal margin of antennal groove slightly above the ventral margin of eye. Eyes small and in lateral view blunt and indistinctly conoidal. Antenna filiform, 14-segmented in male (including scapus and pedicel), while 15-segmented in female, 1.5-2.0 times longer than fore femur. Fastigium of vertex in dorsal view slightly wider than one eye. Fastigium in frontal view between the eyes slightly depressed. Fastigium in dorsal view with well-developed medial and lateral carinae and fossulae. Lateral carina clearly elevated, but in lateral view as high as the dorsal margin of eyes, short, not converging towards the front, bending over at nearly right angles to the medial carina. Transverse carina in dorsal view straight. Frontal costa and facial carina in lateral view minimally visible in front of eye. Tip of fastigium and frontal costa in lateral view slightly, but still clearly, protuberant. Bifurcation of frontal costa into facial carinae between dorsal margin of lateral ocelli and transverse carina, close to transverse carina by less than one diameter of ocellus. Facial carinae in lateral view clearly concave in front of eye and slightly convex in front of antenna. Last segments of maxillary palp not widened. Anterior margin of the pronotum truncated. Pronotum slightly widened between shoulders. Clearly keeled prozonal carinae converging backwards. Median carina distinctly lamellate from anterior margin of the pronotum to the end of tegmen, with a broad depression between shoulders. Interhumeral carinae absent. Internal lateral carinae weak. Pronotum behind shoulders weakly depressed on both sides of median carina, with large black spots like a broad black band (posthumeral). Lateral lobes broadly rounded, more or less close to body. Tegmen almost as long as the fore femur, rather broad, at the end broadly rounded. Macropterous and macropronotal. Alae clearly exceed the pronotum apex. Fore and middle femora short and widened, with wavy margins. Hind femora with very small antegenicular and especially genicular teeth and with some sharply tuberculated raised lobes on the middle outer surface. Posterior tibia distally not or weakly widened, with a few small spines on upper edges. First segment of the hind tarsus not longer than third segment. Pulvilli acute. 


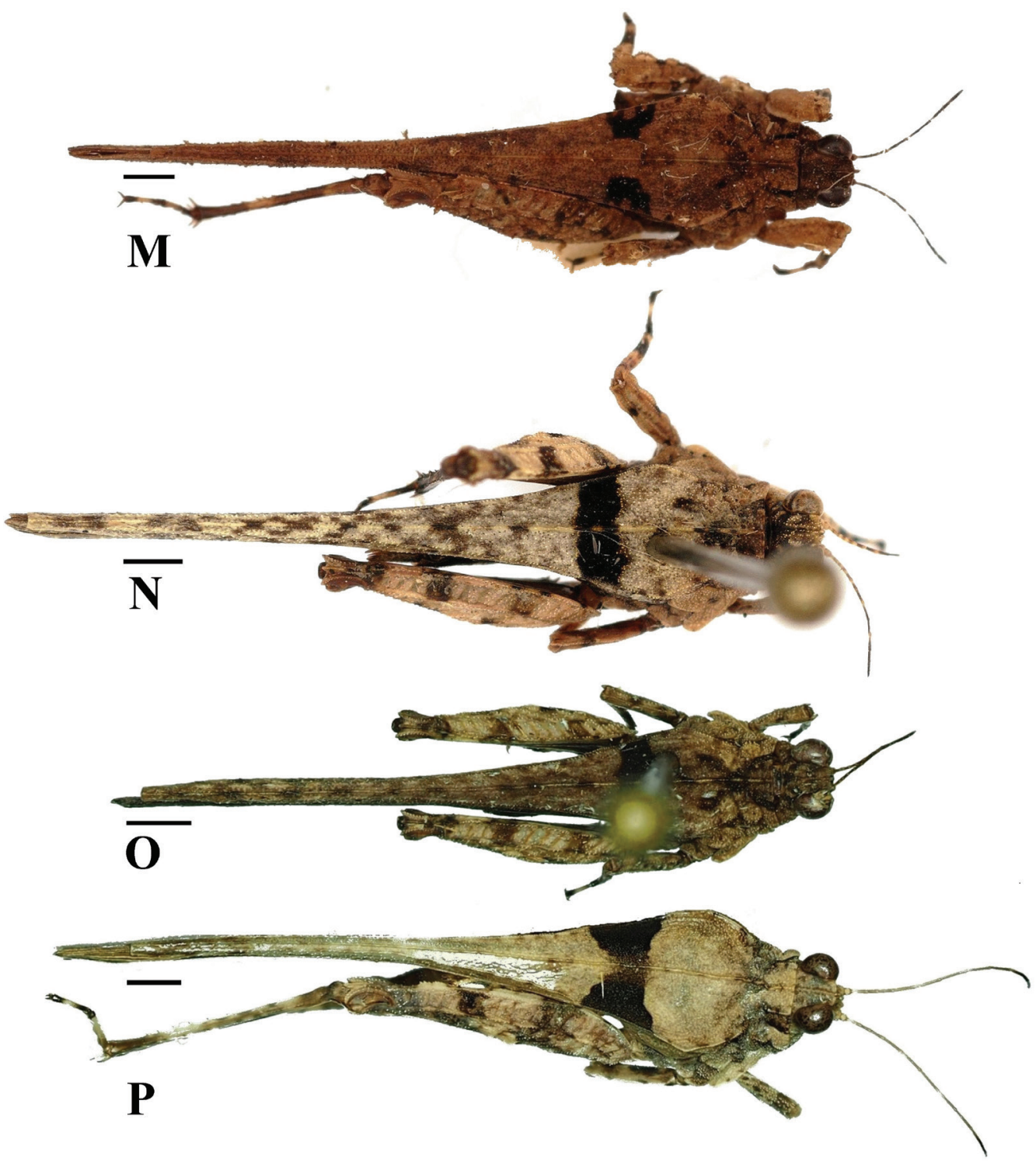

Plate 4. M-P (dorsal view). M. Lamellitettigodes palawanicus (SMTD), + ; N. Lamellitettigodes novaeguineae, ㅇ HT; O. Lamellitettigodes novaeguineae, ठ PT 9/12; P. Lamellitettigodes karwinkeli, q HT. Scale bars: $1 \mathrm{~mm}$.

Measurements. - (in mm) HT: Pronotum length 12.87; pronotum lobe width 3.10 ; pronotum height 1.85 ; vertex width 0.52 ; eye width 0.45 ; tegmen length 1.50 ; hind wing length 10.79; postfemur length 4.68; postfemur width 1.45 .

3 PT $\widehat{o}$ : Pronotum length 9.88-10.40, average 10.18; pronotum lobe width 2.35-2.45, average 2.40; pronotum height $1.3-1.55$, average 1.42 ; vertex width $0.43-0.43$, average 0.43 ; eye width $0.39-0.43$, average 0.41 ; tegmen length $1.20-1.25$, average 1.23; hind wing length 8.97-9.75, average 9.45; postfemur length 4.00-4.10, average 4.05; postfemur width 1.15-1.20, average 1.16.

9 PT우+HT: Pronotum length 10.27-12.74, average 11.62; pronotum lobe width 2.75-3.10, average 2.96; pronotum height $1.65-1.90$, average 1.82 ; vertex width $0.43-0.52$, average 0.49 ; eye width $0.41-0.45$, average 0.43 ; tegmen length $1.30-1.65$, average 1.51; hind wing length 9.36-11.96, average 10.88; postfemur length $4.30-4.75$, average 4.55; postfemur width 1.30-1.50, average 1.38.

Pictures of HT and PT are available in higher resolution in OSF (Cigliano et al. 2019).
Distribution.-The species is only known from lowlands in the South of New Guinea (surroundings of Fly River and Lakekamu River).

Etymology.-The species is named after the island of New Guinea (Nova Guinea) where it is an endemic species of the genus Lamellitettigodes. The specific epitheton is made of two words, adjective and noun of the first (A) Latin declension, both in Genitive case (Novae Guineae = novaeguineae).

\section{Lamellitettigodes karwinkeli sp. nov.}

http://zoobank.org/85026102-A4E2-412C-9310-A8432766EED2

Plates $2 \mathrm{H}, 4 \mathrm{P}, 5 \mathrm{X}$

Material examined.-HT Lamellitettigodes karwinkeli: ${ }_{+}$, China, SYunnan, Xishuangbanna, 23 km NW Jinghong, vic. NA Ban Village (NNNR), $22^{\circ} 10^{\prime} \mathrm{N}, 100^{\circ} 39^{\prime} \mathrm{E}, 700-1000 \mathrm{~m}$, V.-VII.2009 (diverse traps), leg. L. Meng, NMNG. 


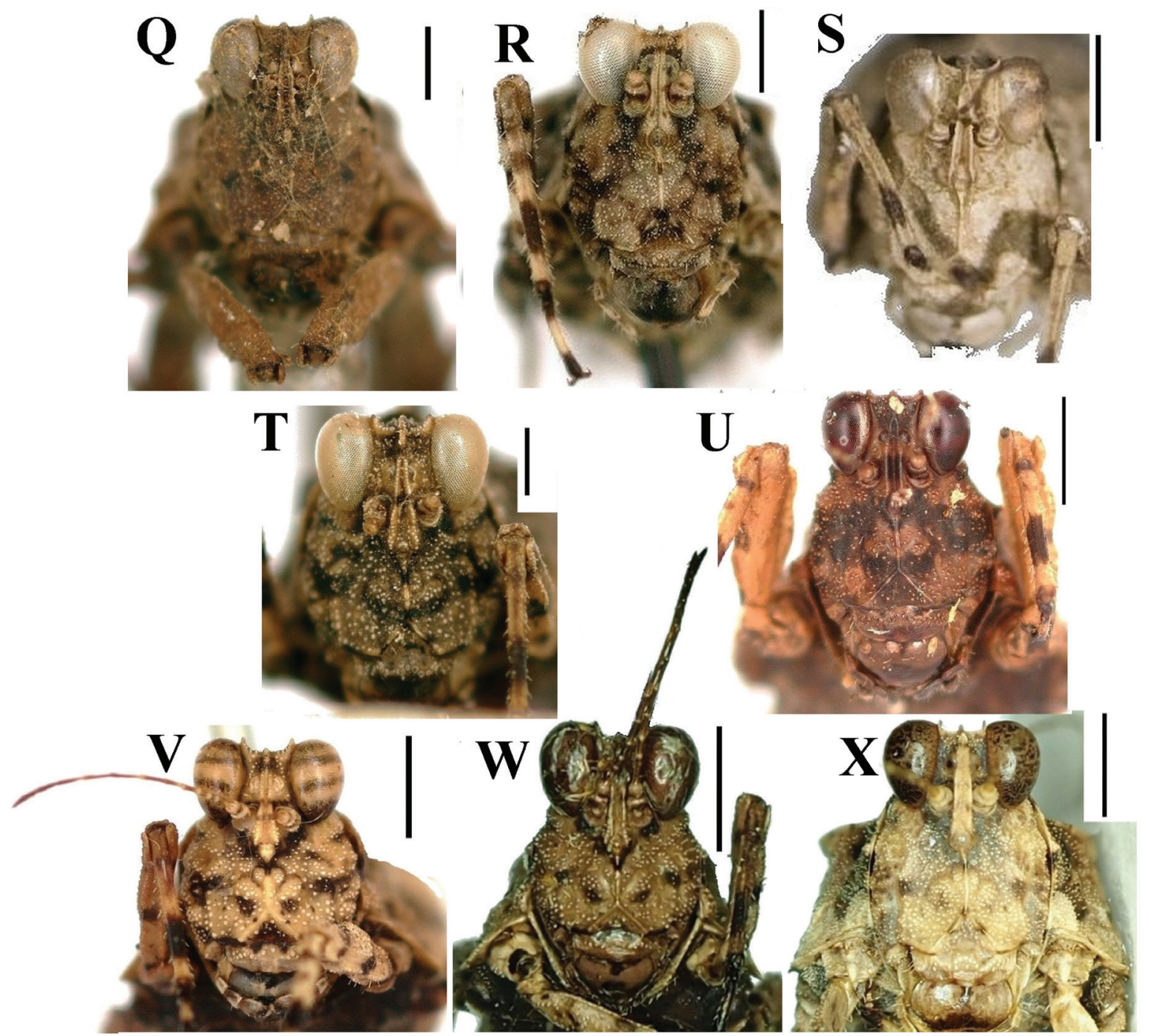

Plate 5. Q-X (frontal view). Q. Lamellitettigodes contractus, + + HT; R. Lamellitettigodes sagittatus, + HT; S. Lamellitettigodes signatus, + HT; T. Lamellitettigodes cultratus, ㅇ HT; U. Lamellitettigodes palawanicus (SMTD), ㅇ; V. Lamellitettigodes novaeguineae, ㅇ HT; W. Lamellitettigodes novaeguineae, §̂ PT 9/12; X. Lamellitettigodes karwinkeli, + HT. Scale bars: $1 \mathrm{~mm}$.

Diagnosis. - L. karwinkeli, together with L. sagittatus and L. signatus species, has pulvilli with apical teeth. Together with L. sagittatus it is a species with long and slender antennae (more than 2 times longer than fore femur. It differs from $L$. sagittatus by the higher pronotum with a broad depression between the shoulders and morphology of fastigium; in frontal view the ventral margin of transverse carina form a right angle with the lateral carina in $L$. karwinkeli, while it is rounded in L. sagittatus.

Description.-Body long and slender. Head and pronotum smooth. Head and eye in lateral view clearly elevated above pronotal discus. Dorsal margin of antennal groove between eyes, slightly above ventral margin of eye. Eyes small and in lateral view blunt and indistinctly conoidal. Antenna (4.5 mm) filiform with long and slender segments, 15-segmented in female (including scapus and pedicel), 2.4 times longer than fore femur. Fastigium of vertex in dorsal view smaller than width of one eye. Fastigium in frontal view between the eyes depressed. Ventral margin of transverse carina forming with lateral carina square (right angle). Fastigium in dorsal view with well-developed medial and lateral carinae and fossulae. Lateral carina clearly elevated, but in lateral view as high as the dorsal margin of eye, short and not converging towards front, bending over at right angles to the medial carina. Transverse carina in dorsal view slightly convex. Frontal costa and facial carina in lateral view clearly visible in front of eye. Tip of fastigium and frontal costa in lateral view distinctly protuberant. Bifurcation of frontal costa into facial carinae close to transverse carina. Facial carinae in lateral view clearly concave in front of eye and slightly convex in front of antenna. Last segments of maxillary palp not widened. Anterior margin of pronotum truncated. Pronotum expanded between shoulders. Clearly keeled prozonal carinae slightly converging backwards. Median carina from the anterior margin of the pronotum to the level of the end of tegmen distinctly keeled, with two flattened elevations before and behind shoulders. Interhumeral carinae absent. Internal lateral carinae weak. Pronotum behind shoulders weakly depressed on both sides of median carina with black spots (posthumeral spots) forming broad black band. Lateral lobes broadly rounded, more or less close to body. Tegmen slightly shorter than fore femora, slender, at the end broadly rounded. Macropterous and macropronotal. Alae clearly exceed the pronotum apex. Fore and middle femora slender, with slightly undulated margins. Hind femora with very small antegenicular and especially genicular teeth, with some 
sharply tuberculate raised lobes on the middle outer surface. Posterior tibia distally not or weakly widened, with a few small spines on upper edges. First segment of the hind tarsus not longer than the third segment of tarsus. Pulvilli distinctly acute with apical teeth.

Measurements. - (in mm) HT: Pronotum length 15.05; pronotum lobe width 3.50 ; pronotum height 2.15 ; vertex width 0.47 ; eye width 0.56 ; tegmen length 1.65 ; hind wing length 12.61 ; postfemur length 6.11; postfemur width 1.43.

Pictures of HT are available in higher resolution in OSF (Cigliano et al. 2019).

Distribution.-The species is only known from the type locality near Xishuangbanna (Yunnan, PR China).

Note: I cannot find any other species of Lamellitettigodes from PR China. There are likely to be further species from PR China that should be assigned to Lamellitettigodes, but since there are only drawings and only a few photographs, such work remains for future revisions.

Etymology.-This species is dedicated to Fabian Karwinkel, a great ornithologist and a committed young conservationist from Northrhine-Westphalia. The specific epitheton is second (US) declension Genitive case of the Fabian's Latinized version of the surname (Karwinkelus $=$ karwinkeli).

\section{Probolotettix Günther, 1939}

Probolotettix kevani Blackith \& Blackith, 1987, syn. nov. Probolotettix kevani: Blackith and Blackith 1987: 5-8; Blackith 1990: 89-90; Blackith 1992: 146; Yin et al. 1996: 902; Otte 1997: 57.

Type material. - HT Probolotettix kevani: $\widehat{\jmath}$, Indonesia, Sulawesi Tengah, Ramu Camp, Kolonodale area, 5.II.1980, leg. P. G. Kevan, LEMQ.

PTs Probolotettix kevani: $\hat{\sigma}$, Indonesia, Sulawesi, Minehassa Prov., Dumoga-Bone National Park, I.-III.1985, leg. R. \& R. Blackith, BMNH, NHMUK10924442; $3 \hat{\jmath}, 2$ 우, Indonesia, Sulawesi Utara, Dumoga-Bone National Park, leg. Blackith, NMI, MZB, MNHNP (not seen); + , Indonesia, Sulawesi Utara, Dumoga-Bone National Park, VII.1985, leg. Butlin, NHMUK10924441; + , Indonesia, Sulawesi Utara, Dumoga-Bone National Park, 17.V.-16. VII.1985, leg. Butlin, LEMQ; $\widehat{\jmath},+$, Indonesia, Sulawesi Utara, Dumoga-Bone National Park, 17.V.-16.VII.1985, leg. Butlin, BMNH, NMI (not seen); ㅇ, Indonesia, Sulawesi Utara, Dumoga-Bone National Park, at light, 19.VII.1985, leg. Butlin, RNMH (not seen, probably lost); + , Indonesia, Sulawesi Utara, Dumoga-Bone National Park, 6.VII.1985, leg. Kirk-Spriggs, BMNH.

The HT is a typical E. personatus (elevated head, short prozona, hind tibia with a bright light ring). Therefore, I synonymize Probolotettix kevani with E. personatus. The description and the drawing of the PT in Blackith and Blackith (1987) refer to a typical Lamellitettigodes sagittatus and I was able to examine three PTs in BMNH and one paratype in LEMQ: there is no doubt that they are L. sagittatus and do not belong to a new species or to Euparatettix personatus.

\section{Scelimena Serville, 1838}

\section{Scelimena novaeguineae (Bolívar, 1898)}

Notes. - I published a wrong specimen as LT (Tumbrinck 2018). The male from IRSNB is a PLT. The correct LT is the following specimen: ${ }^{\lambda}$, Indonesia, New Guinea, Haveri [ $\left.9^{\circ} 22^{\prime} \mathrm{S}, 147^{\circ} 32^{\prime} \mathrm{E}\right]$, leg. Loria, vii-xi.1893. It is one specimen of a series of 43 specimens from Haveri. The other 42 are PLT.

\section{Acknowledgements}

My special thanks go to all institutions for graciously lending me specimens from their collections and for the patience they have shown for my research. I especially thank the Zoologische Forschungsmuseum Alexander Koenig in Bonn and their staff for their support and assistance. I am also thankful to Ingrid Bunker for proof reading and Josip Skejo for their intensive revision.

\section{References}

Blackith RE (1988) The Tetrigidae (Orthoptera) of Sri Lanka. Entomologia Scandinavica Supplement 30: 93-109.

Blackith RE (1990) Tetrigidae with partly exposed wings. Insecta Mundi 4: 87-92.

Blackith RE (1992) Tetrigidae (Insecta; Orthoptera) of South-East Asia: Annotated catalogue with partial translated keys and bibliography. Wicklow, Ireland, $248 \mathrm{pp}$.

Blackith RE, Blackith RM (1987) Tridactylids and Tetrigids (Orthoptera) from Sulawesi, Indonesia. Tijdschrift voor Entomologie 130: 1-10.

Bolívar I (1887) Essai sur les acridiens de la tribu des Tettigidae. Annales de la Société Entomologique de Belgique 31: 175-313.

Bolívar I (1898) Contribution à l'étude des Acridiens espèces de la faune indo et austro-malaisienne du Museo Civico di Storia Naturale di Genova. Annali del Museo civico di storia naturale Giacomo Doria Ser. 2, 19: 66-101. https://doi.org/10.5962/bhl.part.9541

Bruner L (1915) Preliminary catalogue of the orthopteroid insects of the Philippine Islands. University Studies, University of Nebraska 15: 195-281.

Cigliano MM, Braun H, Eades DC, Otte D (2019) Orthoptera Species File - online Orthoptera database. http://Orthoptera.SpeciesFile.org [date 28.08.2019]

Deng WA (2016) Taxonomic study of Tetrigoidea from China. PhD thesis, Wuhan, China: Huazhong Agricultural University, 302 pp.

Günther K (1935) Acrydiinen (Orth. Acrididae) aus dem mittleren Ostborneo, gesammelt von H. C. Siebers 1925. Arbeiten über Morphologische und Taxonomische Entomologie aus Berlin-Dahlem 2: 257-263.

Günther K (1936) Phasmoiden und Acrydiinen (Orthoptera) von Holländisch Neu Guinea hauptsächlich aus den Ausbeuten der Herren Docters van Leuwen (1926), van Heurn (1920), P. N. van Kampen und K. Gjellerup (1910). Nova Guinea, Zoologie 17: 323-352.

Günther K (1937) Acrydiinae (Orthopt. Acrididae) von Java, den Kleinen Sunda-Inseln und Nordaustralien. Revue Suisse de Zoologie 44: 121-140.

Günther K (1938) Acrydiinen (Orthoptera, Acrididae) von Neu Guinea hauptsächlich aus den Ausbeuten von Professor Dr. Bürgers (Deutsche Kaiserin Augusta Fluss-Expedition 1912/1913), Dr. E. Mayr (1928), G. Stein (1931) und Miss L. E. Cheesman (1933/34). Nova Guinea (NS) 2: $1-46$.

Günther K (1939) Revision der Acrydiinae (Orthoptera), III Sectio Amorphopi (Metrodarae Bol. 1887, auct.). Abhandlungen und Berichte des Staatlichen Museums für Tierkunde Dresden, A 20 (NF Bd. 1): $16-335$.

Günther K (1941) Revision der Acrydiinenausbeute H. Sauters von Formosa (Orth.). Stettiner Entomologische Zeitung 102: 145-165.

Günther K (1942) Die Acrydiinae der Oxforder Universitäts-Expedition nach Sarawak vom Jahre 1932. Eos 18: 339-346.

Hancock JL (1907a) Orthoptera Fam. Acridiidae. Subfa. Tetriginae. Genera Insectorum 48: 1-79.

Hancock JL (1907b) Studies of the Tetriginae (Orthoptera) in the Oxford University Museum. Transactions of the Royal Entomological Society of London 1907: 213-244.

Hancock JL (1909) Further studies of the Tetriginae (Orthoptera) in the Oxford University Museum. Transactions of the Entomological Society of London 1908: 387-426. https://doi.org/10.1111/j.1365-2311.1909. tb02160.x 
Hancock JL (1910) Third paper on the Tetriginae (Orthoptera) in the Oxford University Museum. Transactions of the Royal Entomological Society of London 1910: 346-365. https://doi.org/10.1111/j.1365-2311.1910. tb01173.x

Hancock JL (1913) Studies of Tetriginae (Acrydiinae) from the Sarawak Museum, Borneo. Sarawak Museum Journal 1:39-54.

Hancock JL (1915) Indian Tetriginae (Acrydiinae). Records of the Indian Museum 11: 55-137.

Kirby WF (1910) A synonymic catalogue of Orthoptera. Vol. III. Orthoptera Saltatoria. Part II. (Locustidae vel Acrididae). The Trustees of the British Museum London, 674 pp.

Lehmann AW, Devriese H, Tumbrinck J, Skejo J, Lehmann GUC, Hochkirch A (2017) The importance of validated alpha taxonomy for phylogenetic and DNA barcoding studies: a comment on species identification of pygmy grasshoppers (Orthoptera, Tetrigidae). ZooKeys 679: 139-144. https://doi.org/10.3897/zookeys.679.12507

Otte D (1997) Orthoptera Species File 6. Tetrigoidea and Tridactyloidea (Orthoptera: Caelifera) and addenda to OSF Vols 1-5. Philadelphia, 261 pp.

Paris M (1994) Catalogo de tipos de ortopteroides (Insecta) de Ignacio Bolívar, I: Blattaria, Mantodea, Phasmoptera y Orthoptera (Stenopelmatoidea, Rhaphidophoroidea, Tettigonioidea, Grylloidea, Tetrigoidea). Eos 69: 143-264.

Pavón-Gonzalo P, Manzanilla J, García-París M (2012) Taxonomy and morphological characterization of Allotettix simoni (Bolívar, 1890) and implications for the systematics of Metrodorinae (Orthoptera: Tetrigidae). Zoological Journal of the Linnean Society 164: 52-70. https://doi.org/10.1111/j.1096-3642.2011.00764.x

Steinmann H (1970a) Check-list of the Tetricidae (Orthoptera) of the Oriental faunal region. Acta Zoologica Academiae Scientarum Hungaricae 16: 215-240.

Steinmann H (1970b) The Tetricidae (Orthoptera) of the Notogea. Opuscula Zoologica 10: 155-164.
Tumbrinck J (2014a) Taxonomic revision of the Cladonotinae (Orthoptera: Tetrigidae) from the islands of South-East Asia and from Australia, with general remarks to the classification and morphology of the Tetrigidae and descriptions of new genera and species from New Guinea and New Caledonia. In: Telnov D (Ed.) Biodiversity, Biogeography and Nature Conservation in Wallacea and New Guinea. Volume II. The Entomological Society of Latvia, Riga, 345-396.

Tumbrinck J (2014b) Wiemersiella gen. nov.: eine neue DornschreckenGattung von Neuguinea (Orthoptera: Tetrigidae, Batrachideinae). Entomologie heute 26: 73-85.

Tumbrinck J (2015) New species of Palaioscaria Günther, 1936 (Orthoptera: Tetrigidae, Batrachideinae) from New Guinea. Sugapa 9: 29-46.

Tumbrinck J (2018) A new species of Thoradonta from New Guinea with some remarks on other Tetrigidae (Orthoptera) taxa from Indo-Australia. Suara Serangga Papua (SUGAPA digital) 11: 27-46.

Tumbrinck J, Skejo J (2017) Taxonomic and biogeographic revision of the New Guinean genus Ophiotettix Walker, 1871 (Tetrigidae: Metrodorinae: Ophiotettigini trib. nov.), with the descriptions of 33 new species. In: Telnov D, Barclay MVL, Pauwels OSG (Eds) Biodiversity, Biogeography and Nature Conservation in Wallacea and New Guinea. Volume III. The Entomological Society of Latvia, Riga, 525-580.

Willemse C (1928) Spolia Mentawiensia: Acrididae (Orthoptera). Journal of the Malayan Branch of the Royal Asiatic Society 6: 1-12.

Willemse C (1930) Fauna Sumatrensis (Bijdrage Nr. 62). Preliminary revision of the Acrididae (Orthoptera). Tijdschrift voor Entomologie 73: 1-210.

Willemse C (1931) Orthoptera, subfam. Tetriginae der Deutschen Limnologischen Sunda-Expedition. Archiv für Hydrobiologie Supplement 9: $43-46$.

Yin X, Shi J, Yin Z (1996) A Synonymic Catalogue of Grasshoppers and Their Allies of the World. Orthoptera: Caelifera. Beijing, 1266 pp.

Zheng Z, Zeng H, Ou X (2011) A review of the genus Euparatettix Hancock (Orthoptera, Tetrigidae) from China with descriptions of two new species. Acta Zootaxonomica Sinica 36: 383-391. 